\title{
Fast and Accurate Power Line Corridor Survey Using Spatial Line Clustering of Point Cloud
}

\author{
Yuchun Huang $(\mathbb{D}$, Yingli Du (1) and Wenxuan Shi *:(D) \\ School of Remote Sensing and Information Engineering, Wuhan University, Wuhan 430079, China; \\ hycwhu@whu.edu.cn (Y.H.); yinglidu@whu.edu.cn (Y.D.) \\ * Correspondence: shiwx@whu.edu.cn
}

Citation: Huang, Y.; Du, Y.; Shi, W.

Fast and Accurate Power Line Corridor Survey Using Spatial Line Clustering of Point Cloud. Remote Sens. 2021, 13, 1571.

https://doi.org/10.3390/rs13081571

Academic Editors: Mohammad Awrangjeb

Received: 30 January 2021

Accepted: 15 April 2021

Published: 18 April 2021

Publisher's Note: MDPI stays neutral with regard to jurisdictional claims in published maps and institutional affiliations.

Copyright: (c) 2021 by the authors. Licensee MDPI, Basel, Switzerland. This article is an open access article distributed under the terms and conditions of the Creative Commons Attribution (CC BY) license (https:/ / creativecommons.org/licenses/by/ $4.0 /)$.

\begin{abstract}
High-voltage and ultra-high-voltage overhead power lines are important to meet the electricity demand of our daily activities and productions. Due to the overgrowth of trees/vegetation within the corridor area, the distance between the power lines and its surroundings may break through the safety threshold, which could cause potential hazards such as discharge and fire. To ensure the safe and stable operation of the power lines, it is necessary to survey them regularly so that the potential hazards from the surroundings within the power line corridor could be investigated timely. This paper is motivated to quickly and accurately survey the power line corridor with the 3D point clouds. The main contributions of this paper include: (1) the spatial line clustering is proposed to accurately classify and complete the power line points, which can greatly overcome the sparsity and missing of LiDAR points within the complex power line corridor. (2) The contextual relationship between power lines and pylon is well investigated by the grid-based analysis, so that the suspension points of power lines on the pylon are well located. (3) The catenary plane-based simplification of 3D spatial distance calculation between power lines and ground objects facilitates the survey of the power line corridor. Experimental results show that the accuracy of safety distance surveying is $5 \mathrm{~cm}$ for power line corridors of all voltage levels. Compared to the ground-truth point-to-point calculation, the speed of surveying is enhanced thousands of times. It is promising to greatly improve both the accuracy and efficiency of surveying the potential hazards of power line corridor.
\end{abstract}

Keywords: power line corridor; safety distance report; point cloud; spatial line clustering; catenary plane

\section{Introduction}

With the rapid development of the economy and society, the demand for electricity continues to increase, and more and more high-voltage and ultra-high-voltage overhead power lines are built to meet the electricity demand of different regions and industries [1,2]. Due to the overgrowth of trees/vegetation within the corridor area, the distance between power lines and its surroundings may break through the safety threshold, which could cause potential hazards such as discharge and fire [3-5]. To ensure the safe and stable operation of the power lines, it is necessary to survey them regularly so that the potential hazards from the growing trees, buildings, etc. within the power line corridor can be investigated timely [6]. Traditionally, an inspection of the power lines mainly relies on manual instrumentation or visual observation, which is dangerous, time-consuming, and labor-intensive. Some power lines span over complex terrain, pass through lakes and mountains, which are difficult for operators to reach [5,7]. Therefore, there is a need to develop more efficient, safe and accurate methods for the inspection of power line corridor.

As a non-contact, long-distance inspection technology, remote sensing can quickly inspect power lines in a wide area, so it has been widely used in power line inspection. The use of remote sensing technology for power line inspection can be based on 
such data as synthetic aperture radar (SAR) images [8,9], optical satellite images [10,11], optical aerial images [12,13], thermal infrared images [14,15], airborne laser scanning points [16-18], and terrestrial scanning points [19]. SAR images record the scattering characteristics of ground objects by actively transmitting microwave signals. They are easily affected by noise and have low resolutions. Besieds, the SAR images are not intuitive and the interpretation is difficult. Inspection based on optical satellite or optical aerial images is easily restricted by weather conditions, and the images cannot provide direct three-dimensional (3D) coordinates to measure the safety distance between power lines and the neighboring ground objects. It requires dense matching and complex $3 \mathrm{D}$ reconstruction from images to restore the $3 \mathrm{D}$ point cloud and measure the distance $[4,20]$. Thermal infrared images are based on the characteristics of infrared radiation of an object, and are generally used for detecting power line components of huge temperature change [21]. The use of terrestrial mobile measurement equipment for patrol inspections could be restricted by the ground objects within the corridor, and cannot move flexibly in areas of mountain forests, lakes, etc. [22].

Instead, the 3D point cloud collected by laser scanning devices can directly obtain the $3 \mathrm{D}$ coordinates of the ground objects by emitting laser to the ground object and recording the reflection from it [23]. It can effectively solve the complex problem of distance measurement in image processing. Qin et al. [7] used a cable inspection robot equipped with LiDAR to collect data of power lines, but the movement of the robot is inflexible in inspecting the whole power line corridor. Due to its low cost and flexible operation, drones or Unmanned Aerial Vehicles (UAVs) have become the commonly used platforms for data collection in geological survey [24], terrain extraction and modeling [25,26], 3D building reconstruction [27], forestry investigation [28], precision agriculture [29], power line inspection [30-32] and so on. The drones, equipped with LiDAR, GNSS and IMU, can obtain the high density and accurate 3D geographical coordinates of object. Through the laser devices mounted on the drones, the 3D coordinates of the ground objects can be directly obtained, and the safety distance between power lines and the surrounding obstacles can be calculated directly if they are classified. Moreover, 3D laser points from drones are not restricted by the geographical environment, and they are suitable for the fast, flexible and large-scale inspection of power lines, which greatly improve the inspection efficiency of power lines.

The current research on drone LiDAR technology for power line corridor survey can be divided into three aspects: power line extraction, suspension point positioning, and corridor survey.

The extraction of the power line point cloud can be done in the 2-dimensional (2D) projection plane, or directly in the 3D space. Liu et al. and Melzer et al. [33,34] took advantage of the horizontal projection of long-distance conductors and used Hough transform to extract the power line points in the 2D projection plane. Yu et al. [35] also adopted the 2D Hough transform to extract power lines after the ground points and vegetation points were filtered out. Wang performed RANSAC (Random Sample Consensus) to extract power line points in the XOY projection plane after removing most of the vegetation points [36]. However, extraction based on the $2 \mathrm{D}$ projection plane is difficult to distinguish the multiple conductors that are spread vertically.

Most studies of power line extraction in 3D space are mainly grid-based or voxelbased [37]. For a large amount of point cloud data, the method of gridding or voxelization can effectively organize the point cloud and reduce the impact of topographical fluctuations. Ma et al. [38] realized the coarse extraction of power line points using the normalized elevation threshold based on the grid. Jwa et al used a voxel-based detector to extract power lines from airborne point cloud [39]. Spatial distribution descriptors based on the voxel were proposed to extract power line and pylon points [6,40,41]. However, these methods cannot deal with the cases with sparse or missing points of conductors that are close to tall trees, buildings, etc. Besides, grid-based or voxel-based approaches may result in object fragmentation, which could lead to many false negative extractions of the power 
line or pylon. Multi-scale analysis and neighborhood analysis can solve this problem, but multi-scale analysis requires a large amount of computation [42]. Some supervised classification methods, such as JointBoost $[43,44]$, random forest [45], etc., can get better classification accuracy of power lines, but they require careful design of feature and elaborate training of the classifiers with lots of samples.

As the support of the power lines, the high-voltage pylon determines the direction of the conductor and is the basis for the power lines segmentation. Li et al. [46] detected pylon by its large height difference, which could be affected by the tall trees. Peng et al. [47] designed density and slope features to locate pylon, but it would be affected in areas with large slopes. The extraction of pylon is easily disturbed by tall trees and other high buildings. Liu et al. [48] realized the extraction of the pylon by judging the existence of the conductor next to the pylon, but the tall trees near the power lines would still be extracted by mistakes. As the intersecting point of two adjacent segments of power lines, the suspension point is the key to segmenting and fitting the catenary plane of power line points and the subsequent calculation of the safety distance. Yu et al. and Nardinocchi et al. [16,35] located the suspension point at the maximum height of the local area, which was easily affected by the uneven topographical terrain. Shi et al. [49] fitted the 3D line and iteratively computed the intersection in the local area on both sides of the pylon to locate the suspension point. However, it could not deal with the case where the power line point was missing on one side.

The power line corridor survey can be divided into two aspects: clearance anomaly detection and corridor safety distance report. The clearance anomaly detection measures the distance between power lines and the surrounding non-power-facility objects in the corridor, such as trees and buildings, to judge whether the clearance is within the safe range [2]. Chen et al. [40] divided the conductor into sections, and set up a buffer zone to find the abnormal points in the corresponding section. Toschi et al. [50] constructed a balanced k-d tree for the power line points to speed up the search of the anomaly points within the conductor buffer zone. Pu et al. [32] established the spatial indexing with Octree for power line points. Based on clearance anomaly detection, only the ground points that exceed the safety threshold are found. However, to constantly monitor the entire power line corridor, the safety distance report of all the ground points in the corridor is necessary.

For the millions of power line and ground object points, calculating the point-topoint distance is time-consuming. Conductor fitting can help to improve the efficiency of the calculation of safety distance. Chen et al. [17] used the centroid method to sample the conductors for fitting them efficiently, which could reduce the amount of calculation. The mobile fitting method adopted by $\mathrm{Xu}$ [51] is not applicable to the power line with sparse points. An inner-span line analysis based on the MDL (Minimum Description Length) framework was proposed to solve the under- and over- reconstructed conductor model [52]. However, the above fitting methods may perform a good reconstruction of the conductors, but they are not convenient to calculate the safety distance within the power line corridor. Because it is not easy to calculate the distance from a spatial point to a $3 \mathrm{D}$ spatial curve $[53,54]$. In fact, the power line points are located in a vertical plane, which remains not yet exploited in the literature for surveying the 3D distance between the conductor and its surroundings.

Therefore, due to the huge amount of 3D point cloud collected by UAV, as well as the complex terrain within the power line corridor, it remains challenging to accurately and efficiently calculate the distance between the ground objects and power line points. The main challenges include:

(1) The amount of point cloud is huge, making the point-by-point calculation of the distance between millions of power line points and the surrounding ground points prohibitive. Moreover, for the 3D spatial catenary curve of the conductor, it is complicated to calculate the point-to-curve distance directly in the 3D space.

(2) The geographical environment within the power line corridor is complex, which brings difficulty to the accurate extraction of power lines. It spans over mountains, lakes, and terrains with great elevation fluctuation. Moreover, the tall trees could also 
occlude the collection of power line points, resulting in the sparsity or missing points along the power lines.

\section{Methods}

This paper proposes a method to accurately extract power lines from the UAV point cloud and calculate the corridor safety distance at a high speed. As shown in Figure 1, the proposed method included four steps: power line extraction, power line completion, power line segmentation and corridor safety analysis.

(1) Power line extraction: Due to the different height distribution of LiDAR points of power lines, pylons and ground objects, the height histogram was adopted to extract power line points based on gridding. With the help of region growing, the extraction result was further refined by eliminating the false positives.

(2) Power line completion: To complete the missing power line points, spatial line clustering was proposed to accurately classify the power lines from the perspective of projection. For each cluster of the power line, a local linear model was fitted for completing the false negatives.

(3) Power line segmentation: The direction of the power lines varied with the terrains between consecutive pylons, so we needed to treat each span separately. Therefore, the pylon was located by the contextual relationship between power lines and pylon first. The suspension point of conductor on the pylon was then located by progressively searching the nearest intersection of power lines. As a result, the power line points were ready to be segmented span-by-span.

(4) Corridor safety analysis: In order to calculate the distance between ground objects and the power lines, checking the 3D distance between ground points and the power line points in the 3D space was necessary. The points of the power line were distributed as a catenary curve in a vertical plane where the power line points could be fitted first. Then the distance could be simplified using the distance between a 3D spatial point and the catenary plane and the distance between a planar point and a 2D catenary curve.

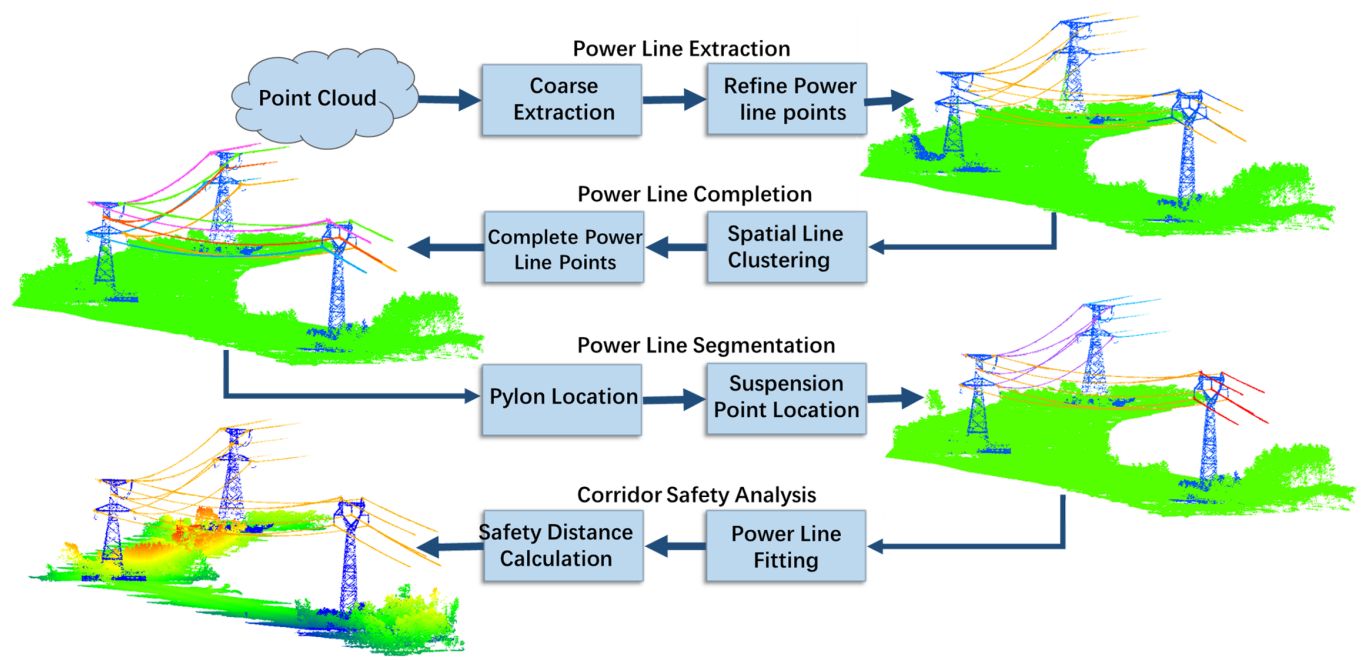

Figure 1. Overview of the method.

Taking full advantage of the contextual and structural distribution of power lines, a 3D distance calculation method based on spatial line clustering and catenary plane is proposed in this paper. The main contributions to the survey of the power line corridor include:

(1) The spatial line clustering is proposed to accurately classify and complete the power line points locally, which can greatly overcome the sparsity and missing of LiDAR points within the complex power line corridor. 
(2) The contextual relationship of the power line and pylon is well investigated by the grid-based analysis, so that pylon and the suspension point of power lines on the pylon are well extracted.

(3) The catenary plane-based simplification of 3D spatial distance calculation between power lines and ground objects facilitates the survey of the power line corridor.

\subsection{Extraction of Power Line Points}

The terrain of the long-distance power line corridor is complicated, and may involves hills, lakes, mountains, etc. The elevations of power lines, pylons and ground objects at different locations of the corridor vary greatly. Therefore, the grid-based local analysis was conducted to convert the large-scale point cloud into organized small-scale cells, which could not only facilitate the organization of point cloud data, but also adapt to terrain fluctuations. The coarse extraction of power lines and pylons was performed by checking the vertical distribution of points in the cell, then the fine extraction of power line points was achieved by combining the neighborhood information of the cell.

\subsubsection{Coarse Extraction of Power Line Points}

According to the $X Y$ coordinates, the point cloud was projected into cells on the $X-Y$ plane. The size of the cell was equal to the size of the pylon planimetric footprints. As shown in Figure 2, among the point cloud of the power line corridor, three blue cuboid boxes correspond to the cuboid containing pylon, power line and ground object, respectively are presented from left to right. The height of the cell containing the pylon was very high $(20-80 \mathrm{~m})$ and the points were continuously distributed along the vertical direction. The height of the cell containing the power lines was also relatively high, but it became empty at some elevation because of the suspension of power lines. Although the cell containing the ground object was also continuously distributed along the vertical direction, the height was relatively low. Since the vertical distribution of points of different objects within the power corridor was quite different, coarse extraction of power lines and pylons could be performed based on the height histogram within each cell.

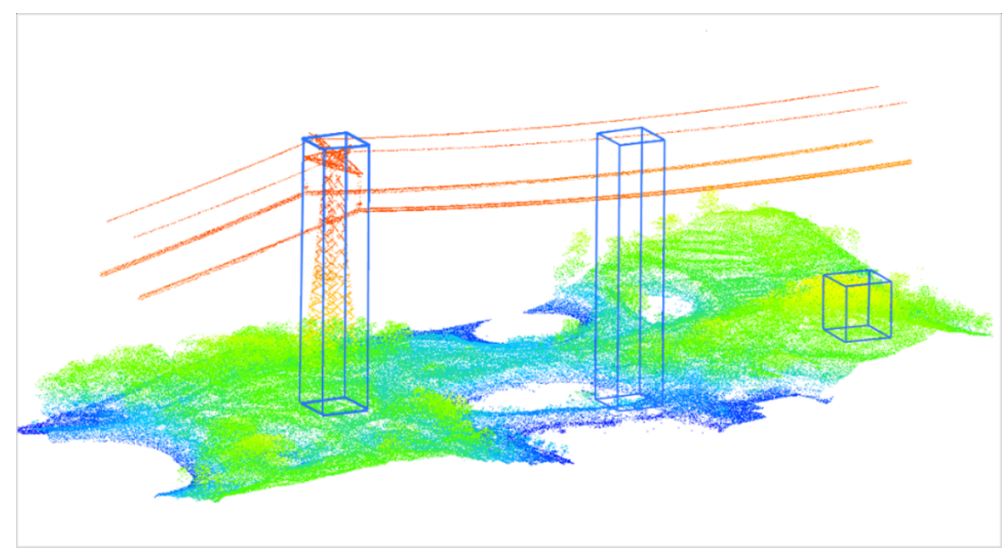

Figure 2. Cells of pylon, power line and ground object.

As shown in Figure 3, it represents the height histogram of the pylon, power line and ground. The height difference of the point cloud in each cell is calculated first. If the height difference is less than the height threshold, which is the minimum distance between power lines and ground, the points in the cell are assumed to be ground. Otherwise, the cell is layered equally along the vertical direction. From the bottom to the top, it is counted whether there is an empty layer. If there is an empty layer, it is considered to be a cell containing power lines. The points above the empty layer are classified as power line points, and the points below are ground points. If there is no empty layer and the height is higher than the height threshold of pylon, it is considered to as pylon, and the points in the cell are all extracted as pylon temporarily. Otherwise, the points were classified as ground. 

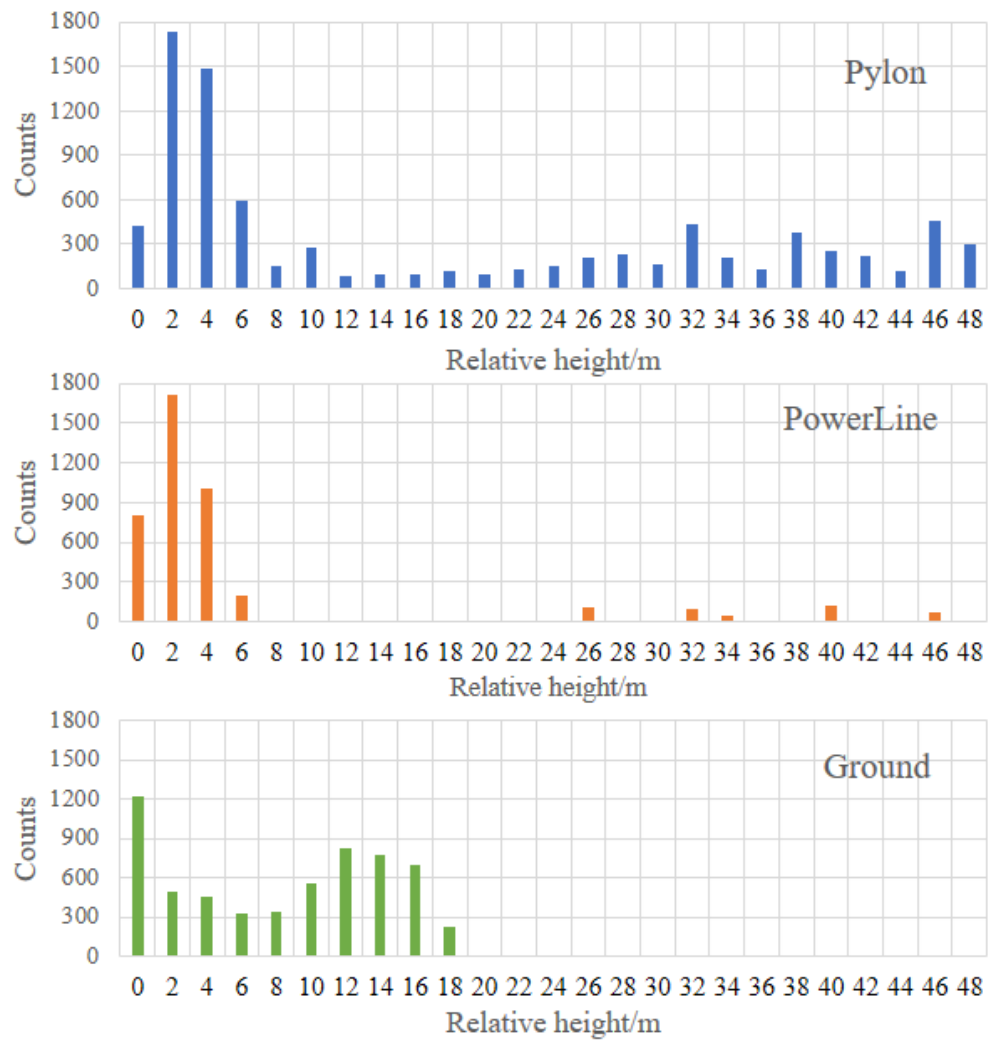

Figure 3. The vertical distribution of a pylon, power line and ground cell respectively.

Figure 4 shows the preliminary result of the candidate points of pylon, power line and ground objects. They are colored in blue, orange and green, respectively. It can be seen that most of the points were effectively extracted. However, there were still a few mis-extracted points because an object might have been divided into two cells by gridding. The red circle A in Figure 4 shows the missing power line points because the power lines were too close to the nearby trees. Because of the false empty layer, the scattered orange points in red circle B were extracted as power line points mistakenly. The blue rectangular C shows the pylons, where some power line points were extracted as pylons mistakenly. The blue rectangular box D shows a wrongly extracted pylon because it was similar to the height of the pylon.

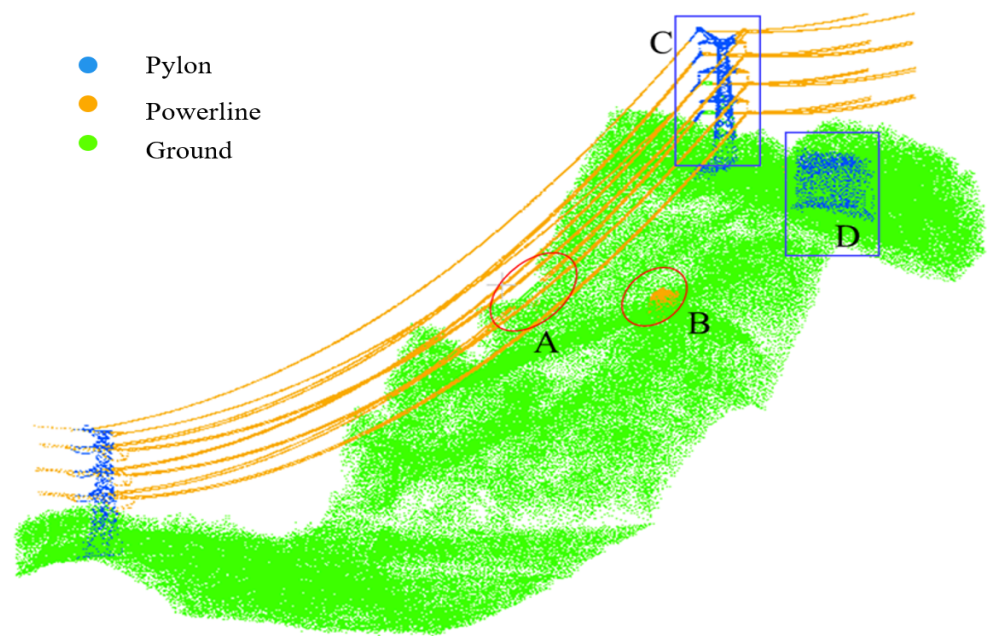

Figure 4. The coarse extraction of pylon and power line points. 


\subsubsection{Refinement of Candidate Power Line Points}

When gridding the point cloud, the outermost canopy of some trees and pylons could be divided into multiple cell segments, as shown in Figure 5a. The orange points were extracted as false positive power line points in the coarse extraction. They could be refined through the following grid-based region growing.

The $3 \times 3$ neighboring cells of a candidate power line cell are illustrated in Figure 5a, where the orange points in the central red cell were extracted as power line points mistakenly. As shown in the top view of Figure 5b, similar to the region growing in a 2D image, we took the non-power line points in the eight neighborhoods as the seeds, and grew them according to the 3D spatial Euclidean distance. If they were grown together with the surrounding points of trees, points in the center cell were re-extracted as non-power line points. Figure $5 \mathrm{c}$ shows the result after region growing, where the mis-extracted power line candidates are corrected as points of trees.

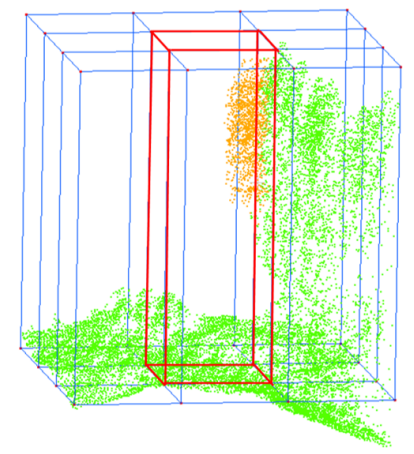

(a)

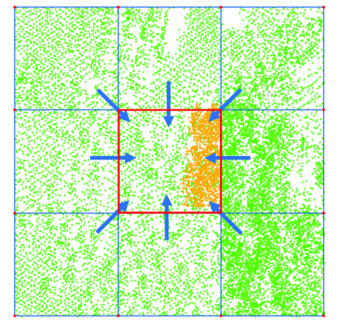

(b)

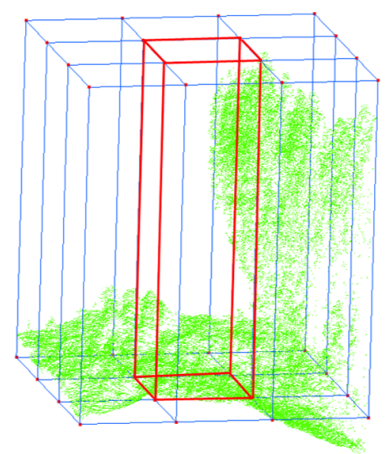

(c)

Figure 5. Removal of false positive power line points using region growing. (a) The $3 \times 3$ neighborhood cells (blue cells) of candidate power line points (orange points in the central cell). (b) The top view of (a) to illustrate the region growing that proceeds inwards along with the blue arrows. (c) The result after region growing, where the candidate power line points are corrected as points of trees.

\subsection{Completion of Power Line Points Using Spatial Line Clustering}

\subsubsection{Cases of Missing Power Line Points}

Although some false positive power line points were eliminated with region growing, other cases of missing or false negative power line points, such as confusion with the pylon, interference from the ground objects and underlying water still existed.

(1) Confusion with the pylon. After the region growing, part of the correctly extracted power line points were lost. As shown in Figure 6a, the power line points near the pylon were extracted as the pylon after the region growing, because they were reachable from the neighboring pylon.

(2) Interference from the ground objects. As shown in Figure 6b, the trees were very close to the power line and lie in the same cell with it, which had an impact on the vertical distribution of the cell, resulting in the lost of the power line in the circles of Figure 6b.

(3) Underlying water. Due to the mirror reflection, there were no laser points from the water surface, which led to another false negative of power line points when power lines passed across the water surface. As shown in Figure 6c, for the cell located at the water, there was no point in the water surface. Since the empty layer between the ground and power line could not be found, the extraction of the power line could not be achieved. 


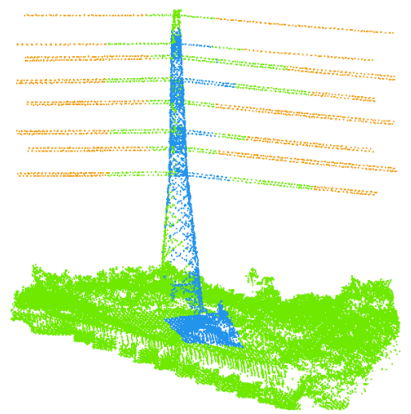

(a)

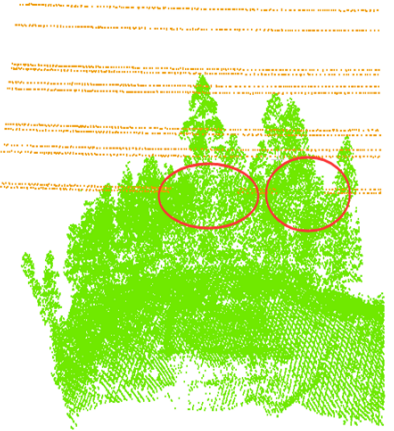

(b)

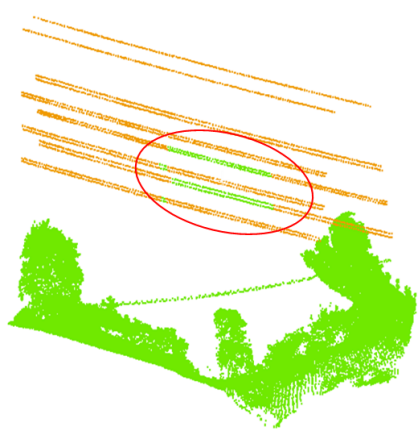

(c)

Figure 6. Typical cases where power line points need to be completed. (a) The power line points near the pylon are classified mistakenly after region growing. (b) The power line points close to the ground objects are occluded, resulting in the missing of power line points in the red circle. (c) The green points in the red circle are lost because of the mirror reflection on the water surface.

\subsubsection{Power Line Classification Using Spatial Line Clustering}

Generally, there were multiple parallel conductors in a span between pylons, so we needed to classify different conductor points before fitting them. Although the conductor was usually bundled by multiple sub-conductors, we treated the bundled conductors as one line to fit and calculated the distance. Because the sub-conductors were very thin and close to each other, it was difficult to identify the individual conductors in the bundle. The simplified power line points of $3 \times 3$ cells are shown in Figure 7a. The points $P_{1}$ and $P_{3}$ belonged to the same line while the point $P_{2}$ belonged to another line. However, the distance of $P_{1} P_{2}$ was smaller than $P_{1} P_{3}$, because the power line points were sparse in some areas. It made it hard to cluster the power line points using the traditional clustering method.

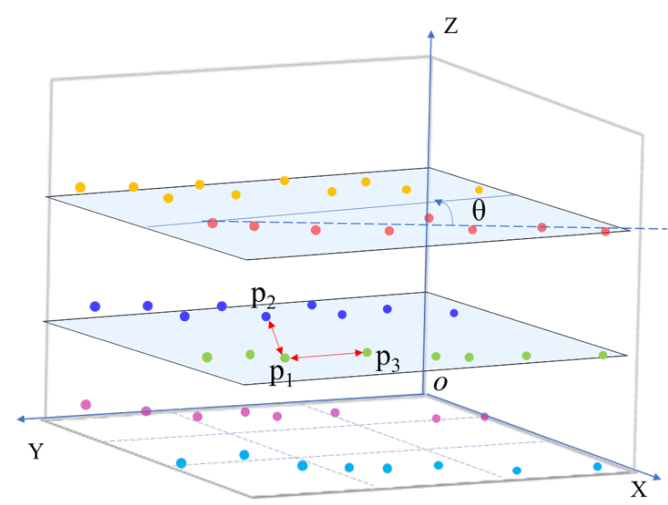

(a)

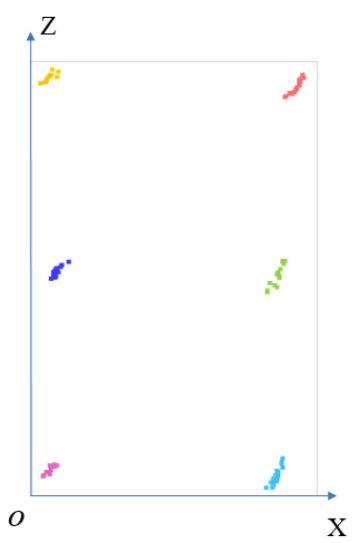

(b)

Figure 7. Spatial line clustering illustration of the power line points. (a) The power line points before projection. (b) The power line points are clustered together after projection.

Since the conductors in the local area were approximately horizontal, we could project the power line points into the plane perpendicular to the direction of the conductor. As shown in Figure 7a, assuming $\theta(0<\theta<\pi)$ was the angle between the conductor and the Y-axis, we could rotate the power line points by angle $\theta$ horizontally, and then project the power line points along the Y-axis into the XOZ plane. As shown in Figure $7 \mathrm{~b}$, the points belonging to the same conductor converged into a cluster in the projected plane, while the points of different conductors were dispersed into different clusters. 
Let $W_{1}$ represent the rotation matrix corresponding to the $\theta$, and $W_{2}$ represent the projection matrix that projects the $3 \mathrm{D}$ point along the $\mathrm{Y}$-axis into $\mathrm{XOZ}$ plane. The whole transformation $W$ can be expressed as the product of the two transformations:

$$
W=W_{2} W_{1}=\left[\begin{array}{lll}
0 & 1 & 0 \\
0 & 0 & 1
\end{array}\right]\left[\begin{array}{ccc}
\cos \theta & -\sin \theta & 0 \\
\sin \theta & \cos \theta & 0 \\
0 & 0 & 1
\end{array}\right]=\left[\begin{array}{ccc}
\sin \theta & \cos \theta & 0 \\
0 & 0 & 1
\end{array}\right]
$$

Let point $P\left(P_{x}, P_{y}, P_{z}\right)$ represent the 3D coordinate of the power line points before projection, and $p\left(p_{x}, p_{z}\right)$ be the $2 \mathrm{D}$ coordinate of the power line point after projection, which is obtained with matrix $\mathrm{W}$. The formula is as follows:

$$
p=\left[\begin{array}{l}
p_{x} \\
p_{z}
\end{array}\right]=W P=\left[\begin{array}{ccc}
\sin \theta & \cos \theta & 0 \\
0 & 0 & 1
\end{array}\right]\left[\begin{array}{l}
P_{x} \\
P_{y} \\
P_{z}
\end{array}\right]
$$

Once the power line points were transformed with the formula of Equation (2), the distribution of the points belonging to the same conductor became more concentrated, while the points belonging to different conducts were more scattered. The probability that point $j$ belongs to the same line with point $\mathrm{i}$ can be expressed by the Gaussian function as follows:

$$
\mathcal{G}_{\mathrm{i}}(\mathrm{j})=g\left(p_{x_{i}}-p_{x_{j}}, p_{z_{i}}-p_{z_{j}}\right)=\frac{1}{\sqrt{2 \pi} \sigma} e^{-\frac{\left(p_{x_{i}}-p_{x_{j}}\right)^{2}+\left(p_{z_{i}}-p_{z_{j}}\right)^{2}}{2 \sigma^{2}}}
$$

where $\sigma$ denotes the radius of the bundled conductor. As shown in Figure 8a, we used $\mathcal{G}_{i}$ to denote the probability that the points concentrated at the point $i$, and it could be calculated by summing the Gaussian response of all the points around point $i$. The formula is as follows:

$$
\mathcal{G}_{\mathrm{i}}=\sum_{j=1}^{N} \mathcal{G}_{i}(j)
$$

where $N$ is the total number of power line points in $3 \times 3$ cells. The bigger the $\mathcal{G}_{i}$, the greater the probability that point $\mathrm{i}$ belongs to a line. For all the points, we maximized the probability by adding all points' $\mathcal{G}_{i}, i=1 \ldots N$ together as follows:

$$
\mathcal{G}=\sum_{i=1}^{N} \mathcal{G}_{i}
$$

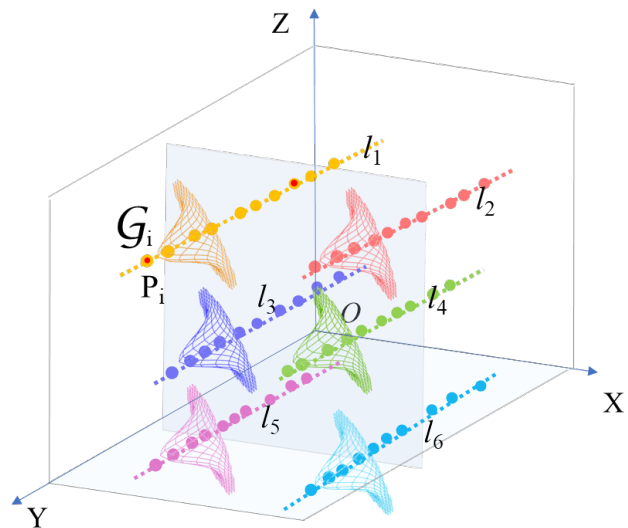

(a)

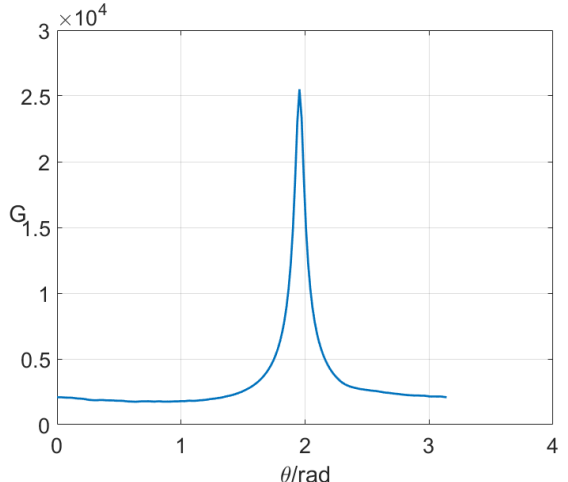

(b)

Figure 8. (a) The process of spatial line clustering. (b) The curve between $\mathcal{G}$ and $\theta$. 
As shown in Figure $8 b, \mathcal{G}$ reached the maximum in the direction of the power line. By solving Equation (6) with the golden section search [55] within the range of $\left[0,180^{\circ}\right.$ ], the direction of power lines $\theta$ could be obtained.

$$
\theta^{*}=\operatorname{argmax} \mathcal{G}
$$

After projection and regularization, we mapped the clusters of power line points into an image using the number of points projected within a pixel as the gray value, as shown in Figure 9a. Figure 9b is the binary image after Ostu thresholding [56] the gray image. After clustering the power line points in the projected image, connected component labeling was employed to locate the clusters of different power lines. As shown in Figure 9c, different color denotes different clusters. After the spatial line clustering, the spatial power line points are denoted with different colors, as shown in Figure $7 \mathrm{~b}$.

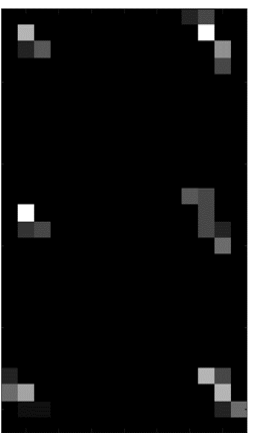

(a)

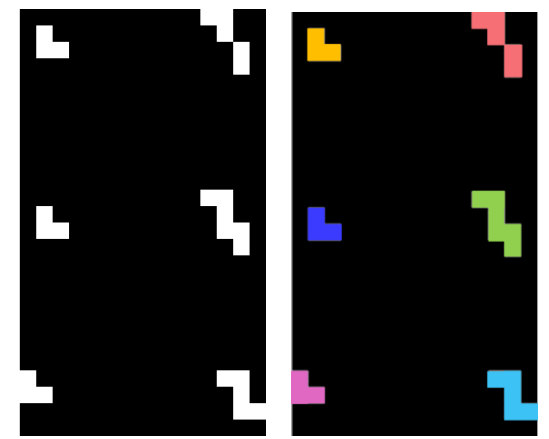

(b) (c)

Figure 9. The clustering process of the power line points. (a) The gray image formed by the projection of spatial line clusters. (b) The binary image after thresholding. (c) Labeling of clusters of conductors.

\subsubsection{Completion of Power Line Points}

Based on the points of a conductor, the equation of the conductor was fitted. Points in the neighborhood whose distance from the fitted line was less than a threshold (e.g., the radius of the conductor) were reclassified as power line points. As shown in the red rectangle A in Figure 10, which is enlarged on the right, the orange dots are the power line points classified correctly in the previous steps, the blue and green dots are the power line points that are classified as pylon mistakenly. The red line $L_{1}, L_{2}$ with the red arrow is the spatial straight line obtained by fitting the cluster of orange power line points. To complete the power line points near the pylon, the distance between point and $L_{1}, L_{2}$ was checked. If the distance was less than the radius of the conductor, it was reclassified as the power line point.

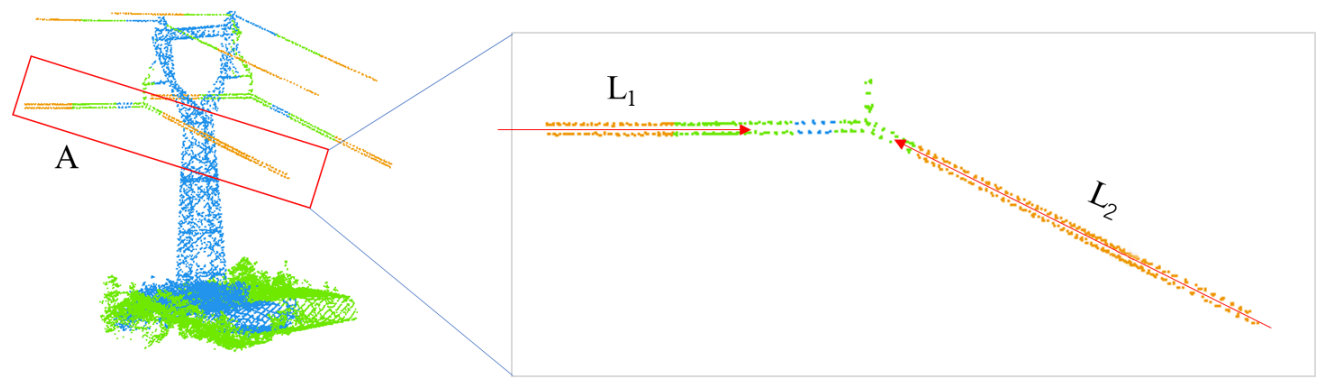

Figure 10. Completion of power line points based on fitting the clusters in the neighborhood. 


\subsection{Power Line Segmentation Using the Suspension Points}

For the long-distance power line corridor, the direction of the power lines between different pylons changed a lot with the terrain, resulting in different catenary equations. It was necessary to segment the power lines into spans by intervals of pylons. Therefore, the pylons were first located in this section, followed by the location of the suspension point on the pylon to segment the power line into spans.

\subsubsection{Pylon Location Guided by Power Line}

During the previous extraction, tall trees that satisfied the height threshold were extracted as pylon mistakenly. We could locate the pylon points with the guidance of power lines so as to remove the false positive pylons. Specifically, for the cell classified as pylon, the distribution of the power line points in its $3 \times 3$ neighborhood cells was analyzed. The pylon cell not surrounded by power line points was considered as a mistake. If there were power line points surrounding it, we needed to see whether the geometric center of the cell was in the convex polygon formed by the horizontal project of surrounding power line points. The pylon cell whose geometric center was in the polygon will be retained.

Figure 11a illustrates the distribution of power lines and pylon after the horizontal projection. The blue-colored cells where $P_{1}$ and $P_{2}$ are located are the pylon cells obtained by the previous extraction. $P_{1}$ and $P_{2}$ are the geometric centers of the corresponding cells, respectively. The cell of $P_{1}$ corresponds to the correct pylon cell, while $P_{2}$ corresponds to the pylon cell extracted incorrectly. The blue dotted rectangle around $P_{1}$ indicates the $3 \times 3$ neighborhood cells and the power line points are marked in orange line. As shown in Figure $11 \mathrm{~b}$, the convex polygons $A_{0} A_{1} \ldots A_{7}$ were defined by the circumscribed convex polygon of the power line points in the $3 \times 3$ neighborhood of $P_{1}$, and polygon $B_{0} B_{1} B_{2} B_{3}$ were defined by the neighbourhood of $P_{2}$ similarly. To judge whether the geometric center $P$ of the pylon cell was within the convex polygon determined by $A_{1} A_{2} \ldots A_{i}$ [57], the following formula (7) was used:

$$
\overrightarrow{A_{i} P} \cdot \overrightarrow{A_{i} A_{i+1}}<0 \quad\left(i=0 \cdots \mathrm{N}-1, A_{N}=A_{0}\right)
$$

where $A_{i}$ is the vertex of the circumscribed convex polygon. If $P$ satisfied Equation (7), it was inside the convex polygon of power lines and regarded as a true pylon. Otherwise, it was removed.

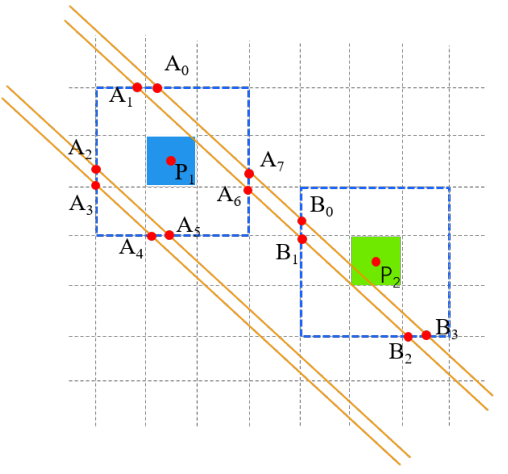

(a)

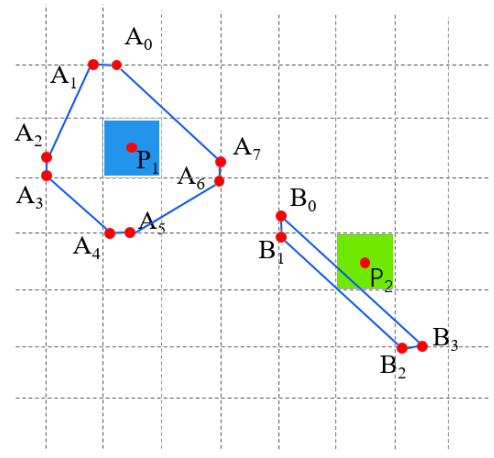

(b)

Figure 11. The removal of the faker pylon. (a) The simplified distribution figure of power lines and pylon after horizontal projection. (b) The convex polygon around the cells of $P_{1}$ and $P_{2}$.

\subsubsection{Suspension Point Location and Power Line Segmentation}

To segment the power lines into spans, the suspension point of the power lines on the pylon was required. The suspension point was the intersection of the power lines on the pylon, and the power lines on different sides of the suspension point belonged to different spans. We took the vertical plane passing through the center of pylon as the initial 
plane to segment the power line. Since the center of the pylon might have been imprecise, the iterative location was performed to get the accurate suspension point. The steps were as follows:

- The horizontal center coordinate of pylon was calculated first. The vertical plane that passed through the center of the pylon and perpendicular to the direction of the power lines was used to segment the power line. As shown in Figure 12, the yellow plane $T_{\text {plane } 1}$ in the first row is a vertical plane passing through the center of the pylon, which acts as the initial interface. It divided the power line points into red and blue parts.

- After fitting the red and the blue points respectively to get $l_{1}$ and $l_{2}$, the intersection of $l_{1}$ and $l_{2}$ was calculated. Since the two lines in the 3D space might have been skew lines which did not intersect, we took the mid-point of the closest point pair of the skew lines as the intersection. As shown in the first row of Figure 12, $p_{1}$ and $p_{2}$ were the closest point pair of the skew lines, and the suspension points $p_{s}$ were the mid-point of $p_{1} p_{2}$. The blue plane was the ideal interface to divide the power lines. It can be seen that the $p_{s}$ were closer to the ideal plane than the original yellow plane.

- If the number of power line points on one side was too small, the intersection of the fitted line on the other side with the plane $T_{\text {plane } 1}$ was directly regarded as the suspension point with no iteration. It often happened when the point cloud was sparse and the power line points were missing on one side.

- If the distance between suspension point $p_{s}$ and the plane $T_{\text {plane } 1}$ was less than a threshold, the calculation stopped. Otherwise, as shown in the second row of Figure 12, it took a new vertical plane $T_{\text {plane2 }}$ passed through the $p_{s}$ to re-divide the power line points, re-fitted the left and right straight lines, and calculated the intersections $p_{s}^{\prime}$. The $p_{s}^{\prime}$ was regarded as the new suspension point. It can be seen that the new suspension point approached the ideal plane gradually.

- The above process was repeated until the distance of successive intersection $p_{s}$ and $p_{s}^{\prime}$ was less than a threshold.

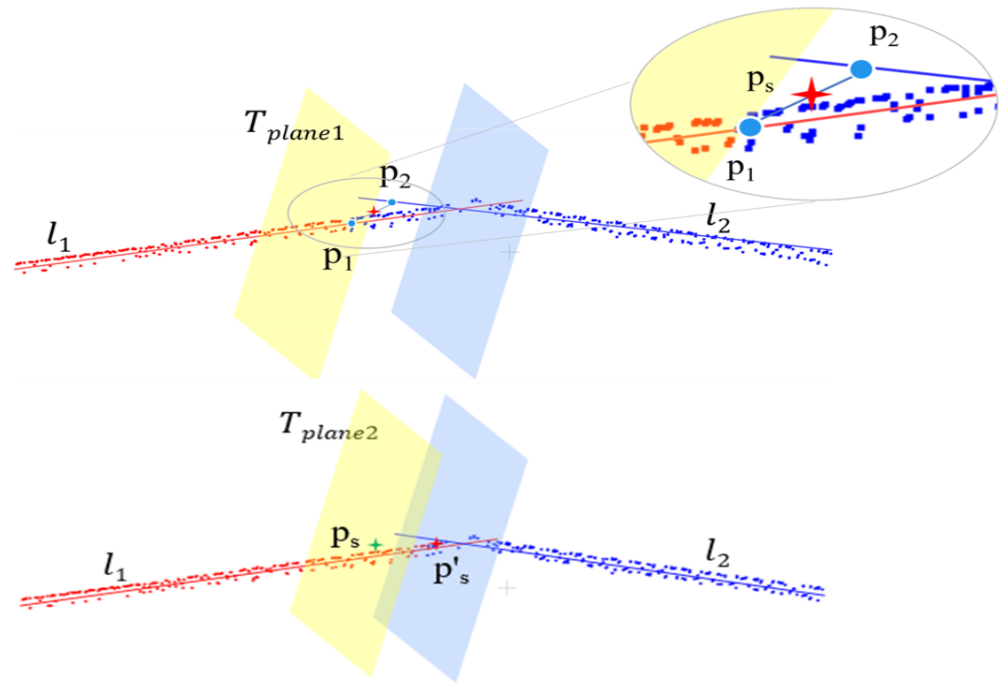

Figure 12. Suspension points location under the guidance of pylon.

\subsection{Power Line Fitting and Corridor Safety Distance Calculation}

To compute the safety distance between ground objects and conductors, checking the spatial distance between ground points and the power line points was required. It was time-consuming to compute the point-to-point distance between the ground objects and the conductors because the number of the power line points was large. Actually, the points of the conduct were distributed as a catenary curve. The safety distance of ground objects could be computed by the distance from the ground point to the threedimensional (3D) catenary curve. However, computing the distance from a point to a 3D 
curve was complicated [53]. Existing research on the distance from a point to 3D curves mainly focuses on such parameterized spatial curve as the NURBS curve, Bezier curve, etc. [58].

Actually, under the conditions of the small influence of wind, the power line points were distributed in a vertical plane due to gravity, which is called the catenary plane. Therefore, the distance from a ground point to a 3D spatial curve of the conductor could be decomposed into the distance between a 3D spatial point and the catenary plane and the distance between a planar point and a 2D catenary curve. To simplify the calculation of the distance between the point and the catenary plane and the fitting of the catenary curve of the conductor, this paper regularized the points by rotating them around the Z-axis and making the catenary plane parallel to a coordinate plane (such as YOZ). The 3D spatial curve of the conductor could be simplified as a 2D curve. As shown in Figure 13a, we took one of the power lines $l_{4}$ as an example to illustrate the process.

\subsubsection{Regularizing the Point Cloud}

As shown in Figure 13a, the blue quadrilateral represents the catenary plane where the power line points were located. The red dotted line is the projection of the conduct, and its direction vector is represented by $\left(v_{1}, v_{2}, 0\right)$. The vector $\left(v_{1}, v_{2}\right)$ could be calculated by taking the Principal Component Analysis (PCA) of the power line points. The angle between power line plane and $\mathrm{YOZ}$ coordinate plane was expressed by $\theta$. Then the relationship between $\theta$ and the direction vector $\left(v_{1}, v_{2}, 0\right)$ was as follows:

$$
\tan \theta=\frac{v_{1}}{v_{2}}
$$

for the point $P(x, y, z)$, its regularized coordinate $P^{\prime}\left(x^{\prime}, y^{\prime}, z^{\prime}\right)$ after rotation can be calculated by rotating $\theta$ as follows:

$$
\left[\begin{array}{l}
x^{\prime} \\
y^{\prime} \\
z^{\prime}
\end{array}\right]=\left[\begin{array}{ccc}
\cos \theta & \sin \theta & 0 \\
-\sin \theta & \cos \theta & 0 \\
0 & 0 & 1
\end{array}\right]\left[\begin{array}{l}
x \\
y \\
z
\end{array}\right]
$$

where $(x, y, z)$ and $\left(x^{\prime}, y^{\prime}, z^{\prime}\right)$ represent the coordinates of the point $P$ before and after the regularization, and $\theta$ is the angle rotating around the $Z$-axis. It can be seen from Figure $13 \mathrm{~b}$ that the conductors became parallel to the $\mathrm{YOZ}$ plane after the regularization.

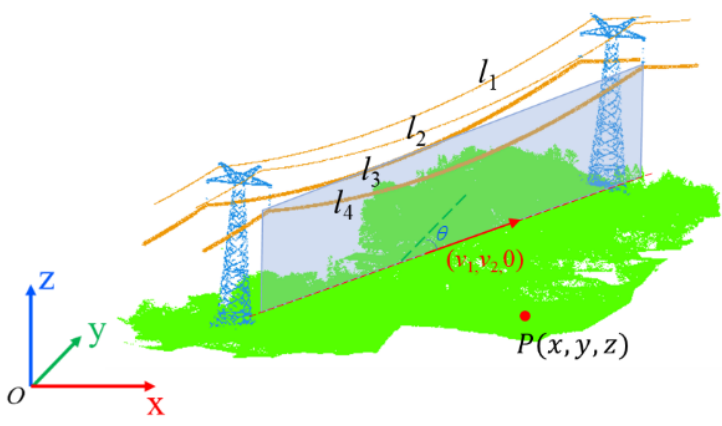

(a)

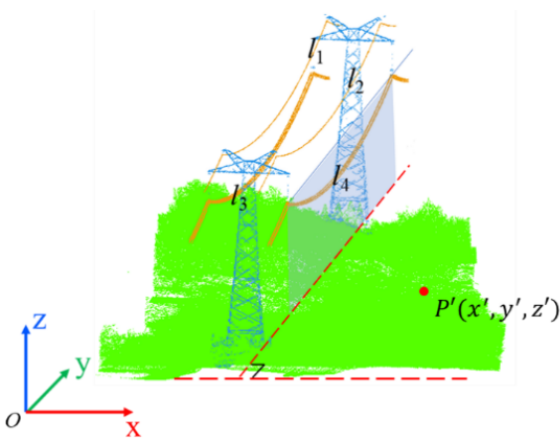

(b)

Figure 13. The point cloud scene before and after regularization. (a) Original point cloud where the direction of the power line plane is irregular. (b) The point cloud after regularization where the catenary plane becomes parallel to the YOZ plane. 


\subsubsection{Fitting the Catenary Plane and Catenary Curve of Power Line}

Since the catenary plane became parallel to the YOZ plane after the regularization, the plane of $l_{i}$ could be represented as the average of the $x$ coordinate of all regularized power line points; the formula was as follows:

$$
\begin{gathered}
x_{i}=\frac{1}{n} \sum_{j=1}^{n} x_{i j}^{\prime} \\
x=x_{i}
\end{gathered}
$$

where $x_{i j}^{\prime}$ represents the $\mathrm{x}$ coordinate of the jth point of the ith conductor after the regularization. $x_{i}$ is the average of the $x_{i j}^{\prime}$. There were four planes corresponding to the $l_{1}, l_{2}, l_{3}$ and $l_{4}$ respectively, so we needed to calculate four planes by Equation (10) and Equation (11). Since the catenary plane was parallel to the YOZ plane after regularization, the catenary curve fitting of the conductor became a 2D catenary fitting. Due to the effect of gravity, the $2 \mathrm{D}$ catenary curve physically met the following catenary equation [22,59]:

$$
z=\operatorname{acosh}\left(\frac{y^{\prime}-b}{a}\right)+c
$$

where $a, b, c$ are the parameters. They can be solved with least-squares by the following:

$$
\underset{a, b, c}{\operatorname{argmin}} \frac{1}{n} \sum_{i=1}^{n}\left[z^{\prime}-\operatorname{acosh}\left(\frac{y^{\prime}-b}{a}\right)+c\right]
$$

\subsubsection{Calculate the Safety Distance}

Based on the catenary curve fitting of power line points, the distance between the conductors and the ground objects within the power line corridor could be calculated. With the cells of the point cloud, the safety distance could be calculated in two steps as follows:

1. For most cells that neither contained any power line points nor any power line points in the neighboring cells, they were safe with no report of safety distance.

2. If the cell had power line points or had a power line in its neighborhood, we needed to calculate the safety distance of every object point in the cell.

As shown in Figure 14, the distance of the ground point $P_{g}\left(x_{g}, y_{g}, z_{g}\right)$ away from the conductor $l_{i}$ could be calculated by Pythagorean theorem after the regularization. $\Delta x_{g}$ is the distance between the ground point and the catenary plane, which is described by Equation (14). $\Delta z_{g}$ is the planar distance between the object point $P_{g}$ and the 2D catenary curve in the catenary plane, which is described by Equation (15). The distance $d_{g_{-} i}$ could be calculated by Equation (16) using Pythagorean theorem:

$$
\begin{gathered}
\Delta x_{g}=x_{g}-\frac{1}{n} \sum x^{\prime} \\
\Delta z_{g}=\operatorname{acosh}\left(\frac{y_{g}-b}{a}\right)+c-z_{g} \\
d_{g_{-} i}=\sqrt{\left(\Delta x_{g}\right)^{2}+\left(\Delta z_{g}\right)^{2}}
\end{gathered}
$$

Taking the minimum distance as the safety distance, we had the safety distance of the ground point as follows:

$$
d_{g}=\min \left(d_{g_{-} 1}, d_{g_{-}}, \ldots d_{g_{-} N}\right)
$$

where $d_{g_{i} i}$ denotes the distance between the ground point $P_{g}$ and $l_{i}, N$ represents the number of conductors in the span ( $N$ equals to 4 in Figure 14). 


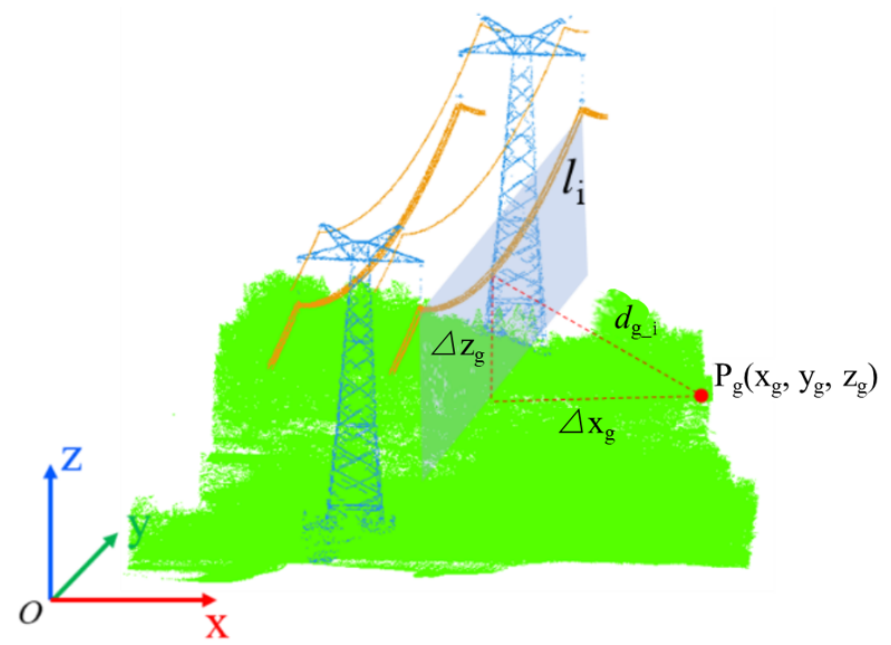

Figure 14. The calculation of safety distance using the catenary plane.

\section{Results}

\subsection{Experimental Data}

In order to test the performance of the proposed method, power lines of three different voltage levels, including $220 \mathrm{kV}, 500 \mathrm{kV}$ and $750 \mathrm{kV}$, were scanned by a Velodyne HDL32 LiDAR mounted on a drone at the height of about $100 \mathrm{~m}$. The scanner could collect 700,000 points per second with an accuracy of $\pm 2 \mathrm{~cm}$ in range measurement. The details of the datasets are listed in Table 1 . They were acquired in different regions, and the corridor terrain covered the steep mountains, large lakes and lush trees. There were two corridors in the data of the $220 \mathrm{kV}$ power line, one is $1.4 \mathrm{~km}$ containing four pylons, and the other was $5.1 \mathrm{~km}$ containing 12 pylons. The density was 63 points $/ \mathrm{m}^{2}$ in average with 36.93 million points in total. For the dataset of $500 \mathrm{kV}$ power line, the corridor length was $21.2 \mathrm{~km}$ with 69 pylons. There were 170.92 million points in total and the density was 89.3 points $/ \mathrm{m}^{2}$. The length of the $750 \mathrm{kV}$ power line corridor was $14.2 \mathrm{~km}$ with 33 pylons. There were 64.13 million points with a density of 49 points $/ \mathrm{m}^{2}$.

Table 1. The description of the three level datasets.

\begin{tabular}{cccc}
\hline & $\mathbf{2 2 0 ~ \mathbf { ~ V }}$ & $\mathbf{5 0 0 ~} \mathbf{~ V}$ & $\mathbf{7 5 0 ~} \mathbf{~ V V}$ \\
\hline Length $(\mathrm{km})$ & 5.1 & 21.2 & 14.2 \\
Number of points (Million) & 36.93 & 170.92 & 64.13 \\
Density (points $\left./ \mathrm{m}^{2}\right)$ & 63.0 & 89.3 & 49 \\
Number of pylons & 16 & 69 & 33 \\
\hline
\end{tabular}

\subsection{Evaluation Methods}

\subsubsection{Evaluation of the Extraction Effect of Power Line Points}

In order to evaluate the effectiveness of the power line extraction quantitatively, we marked power line points manually as ground truth, and power line points extracted by the proposed method were compared with the ground truth. The evaluation method was as follows:

$$
\begin{gathered}
\text { Precision }=\frac{T P}{T P+F P} \\
\text { Recall }=\frac{T P}{T P+F N} \\
F_{\text {score }}=2 \cdot \frac{\text { Precision } \cdot \text { Recall }}{\text { Precision }+ \text { Recall }}
\end{gathered}
$$


where $T P, F P$ and $F N$ represent the numbers of the true positive, false positive and false negative, respectively. It is clear that higher precision meant fewer false positives and higher recall meant fewer false negatives.

\subsubsection{Spatial Positioning Accuracy of the Suspension Points}

For the suspension points, the calculated coordinate of the suspension point $P$ is compared with the manually marked suspension point $P_{g t}$, and the accuracy was evaluated as follows:

$$
\Delta d=\left|\overline{P_{g t} P}\right|
$$

where $P_{g t}$ represents the ground truth obtained by the manual measurement, and $P$ represents the coordinate calculated by the proposed method. The spatial positioning accuracy $\Delta d$ was calculated using the 3D Euclidean distance.

\subsubsection{The Accuracy of the Safety Distance Calculation}

We calculated the distance between the ground points and the power line points point-by-point, and took the minimal distance as the ground truth. In this way, a lot of ground truth could be obtained easily. The mean value of the absolute error (MAE) of safety distance was adopted to evaluate the accuracy of the distance calculated by the proposed catenary-plane-based method.

$$
\operatorname{MAE}=\frac{1}{\sum_{i=1}^{n} m_{i}} \sum_{i=1}^{n} \sum_{j=1}^{m_{i}} a b s\left(d_{i j}^{1}-d_{i j}^{2}\right)
$$

where $\mathrm{n}$ is the number of the spans, $d_{i j}^{1}$ denotes the distance between the jth ground point and the ith conductor calculated by the catenary-plane-based method in this paper, $d_{i j}^{2}$ denotes the ground-truth distance of point $j$, and $m_{i}$ represents the number of the ground points within the corridor.

\subsection{Experimental Results}

\subsubsection{Results of Typical Corridors}

As shown in Figure 15, for a typical scene of $220 \mathrm{kV}$ power lines, the results of intermediate steps, including the classification result, the fitting of the conductors and the safety distance are presented. The raw data were rendered according to the elevation from blue to green to red (Figure 15a).

Firstly, the height histogram analysis was adopted to coarsely classify the power line and pylon points based on gridding (Figure 15b). The effect of ground fluctuation was effectively eliminated by gridding. However, there were also many false positives caused by the gridding, such as the orange points in the red circle and the blue points in the blue rectangle, as shown in Figure 15b.

After the region growing, the coarse extraction was further refined by eliminating the false positives of power line points that were extracted mistakenly. It can be seen that the orange points in the red circle in Figure 15b are removed (Figure 15c). However, the refinement may have led to the false negatives of power line points near the pylon, as shown in the red rectangular $\mathrm{a}$ and $\mathrm{b}$ in the Figure $15 \mathrm{c}$.

To complete the false negative power line points, spatial line clustering was performed to accurately classify the power lines from the perspective of projection. For each cluster of the power line, a local linear model was fitted(Figure 15d), where the missing power line points in the red rectangular $a$ and $b$ were extracted exactly.

Using the contextual relationship between the power line and the pylon, the mistakenly extracted pylon cell (blue points in the blue rectangular of Figure 15b) was removed (Figure 15e). The suspension points were then located by iteratively searching the nearest intersection of power lines on both sides of the pylon, and the whole power lines could be 
thereafter segmented span-by-span. The red points in the enlarged red rectangle are the suspension points (Figure 15f).

To survey the corridor safety, the conductor was fitted with the catenary plane (Figure 15g). The result of the safety distance is shown in Figure 15h. The points close to the conductor are colored with hot red while the points far away from the conductors are colored with cold blue.

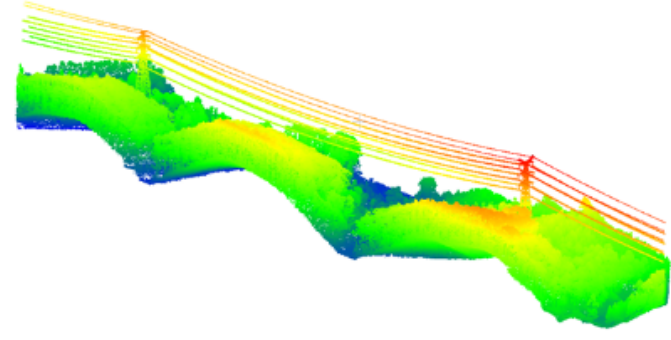

(a)

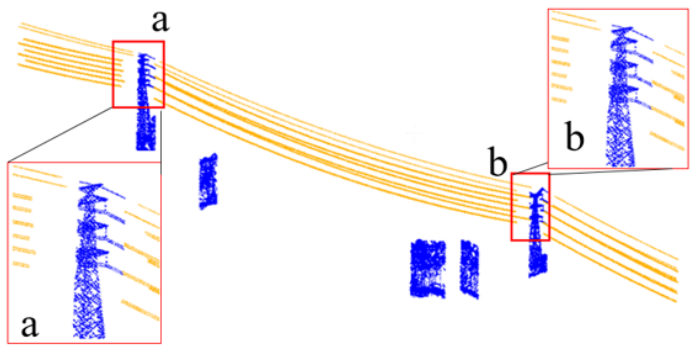

(c)

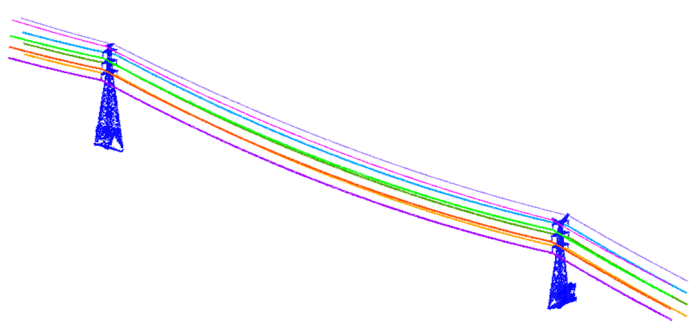

(e)

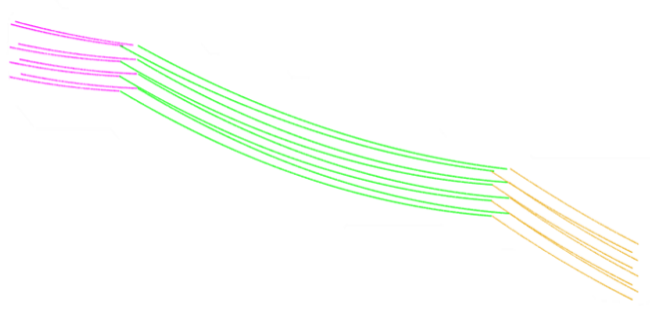

(g)

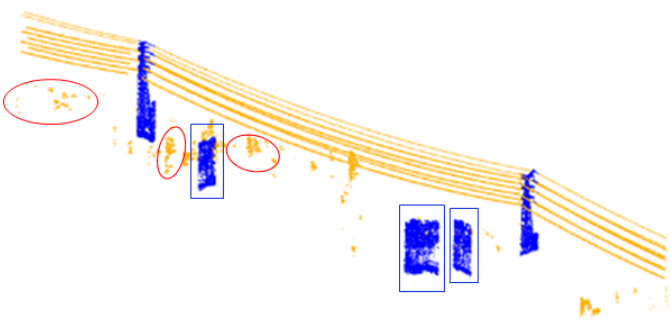

(b)

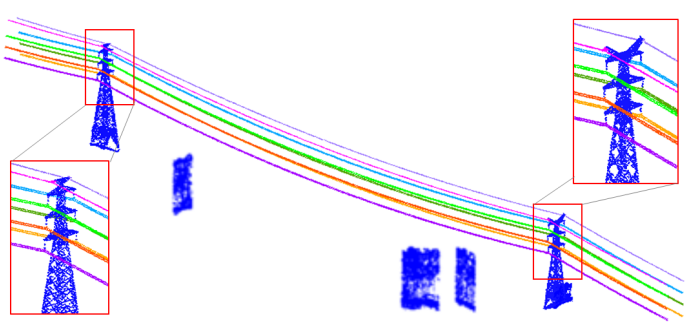

(d)

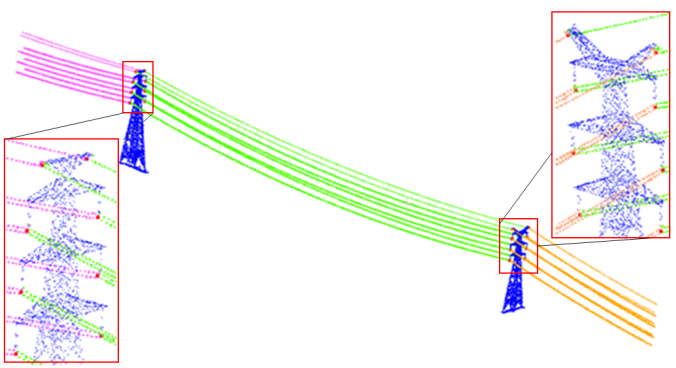

(f)

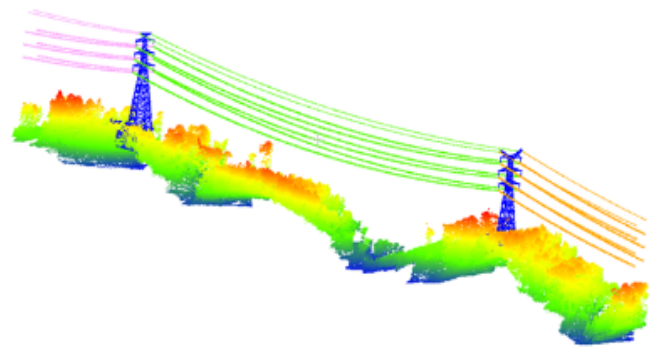

(h)

Figure 15. The process of a typical scene of $220 \mathrm{kV}$ power lines. (a) The original scene of the power lines rendering according to the elevation. (b) The coarse extraction result. (c) The result of removing false positive power line points. (d) The completed result of the missing power line points. (e) The result of removing the false positive pylons. (f) The extraction result of the suspension points and the segmentation result of power lines. (g) The fitting result of the conductors. (h) The result of the safety distance of the ground points. 
Figure 16 shows the scene of power line completion using the proposed spatial line clustering. As shown in Figure 16a, since some power line points were sparse and missing, there were some mis-classified points near the pylons marked in the red circle. With the help of spatial line clustering and conductor fitting, the complete power line points were obtained as shown in Figure 16b, where the power line points and pylons were extracted exactly.

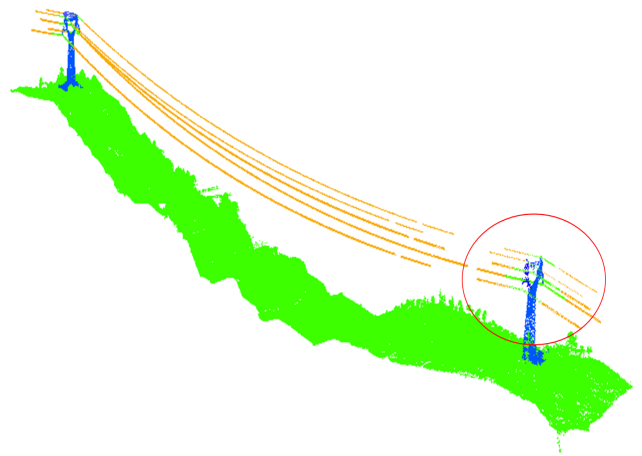

(a)

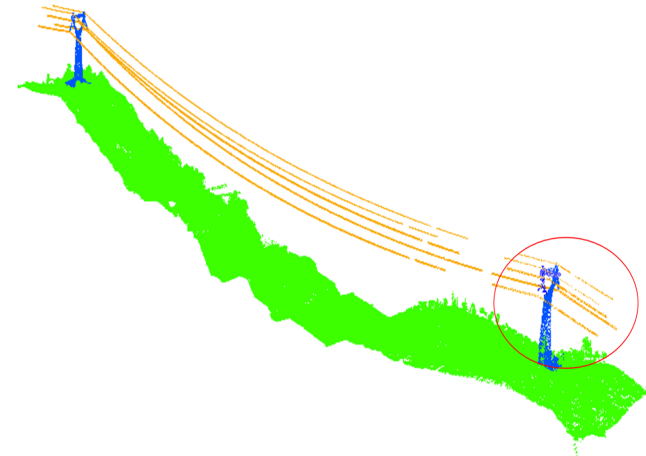

(b)

Figure 16. (a) The coarse extraction result in sparse points area. (b) The results after the spatial line clustering and power line completion.

Figure 17 shows the main extraction process of $220 \mathrm{kV}$ power lines in a large-scale scene. The original scene of the power lines was rendered according to the elevation. From blue to red, it indicates that the heights of the points became higher gradually (Figure 17a). It can be seen that the terrain of this scene could undulating, the height of the pylons varied a lot, and the trees in the local area were relatively lush, even close to the height of the pylon (red circle area in the right bottom). The coarse extraction and false positive removal were performed first, where the orange, blue and green points represent the result of power line, pylon and ground points, respectively (Figure 17b). However, many non-electric pylon points on the ground were mis-classified as pylon and colored with blue. From the enlarged view of the red circle area, it can be seen that the trees and the electric pylon were relatively close in terms of the vertical distribution, resulting in the trees being extracted as pylons mistakenly. By incorporating the spatial contextual relationship between power lines and pylon, a large number of false positive pylon points were removed. It greatly facilitated the positioning of suspension points of power line, which could be seen from the result of the safety distance report of the ground points in Figure 17d, where the power line and pylon points are colored with orange and blue respectively. The ground points from blue to red indicated that the points were getting closer to the conductors.

\subsubsection{The Extraction Accuracy of Power Line Points}

As shown in Table 2, the Precision, Recall and F-score for extracting the power lines of $220 \mathrm{kV}, 500 \mathrm{kV}, 750 \mathrm{kV}$ exceeded $97.85 \%, 98.18 \%$ and $98.02 \%$, respectively, indicating that the algorithm had a good extraction effect for power lines of different voltage levels. The false positives and false negatives were mainly occurred at the junction of the conductor and pylons. Because the pylon points close to the conductor were somewhat confused with power line points when taking power line completion. With the increase of the power line voltage level, the radius of the conductor became larger, and a larger threshold radius needed to be set when taking the power line completion, so the confusion between the pylon points and power line points increased. Thus the precision and recall of $750 \mathrm{kV}$ power lines were slightly smaller than those of $500 \mathrm{kV}$ and $220 \mathrm{kV}$. 


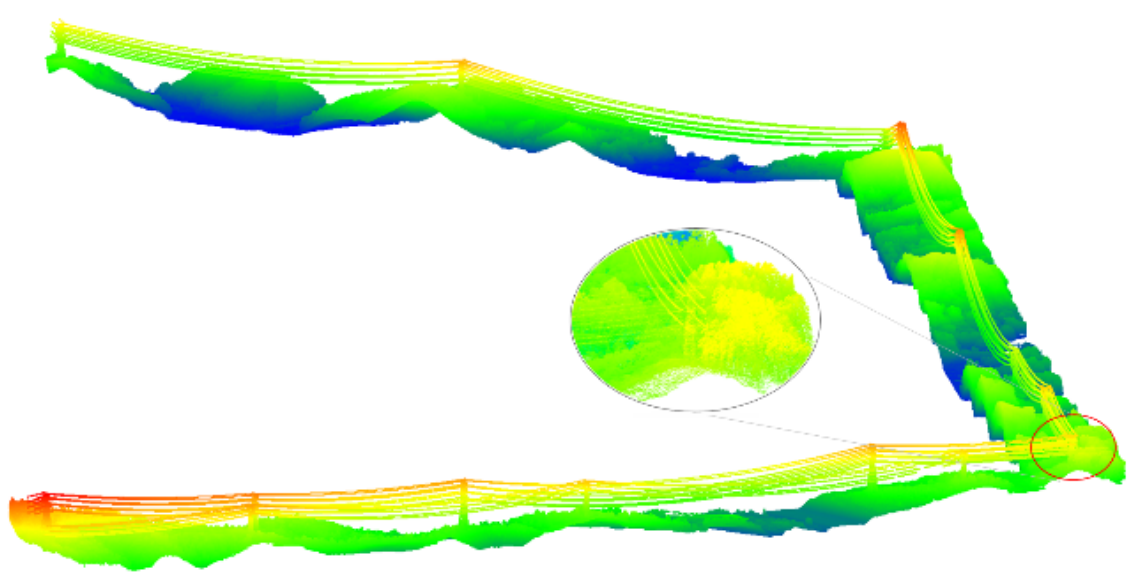

(a)

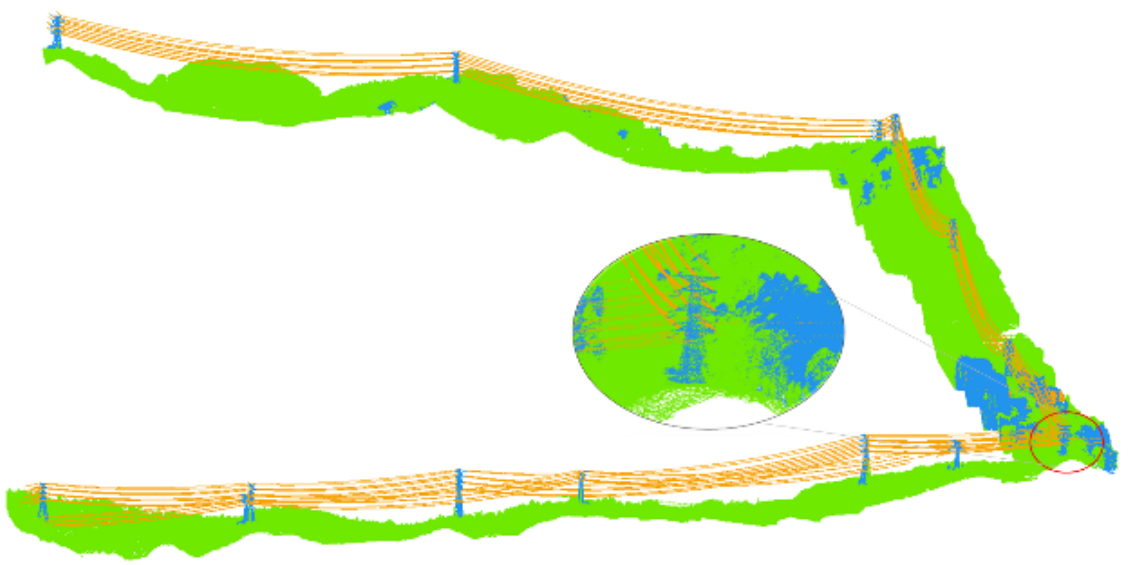

(b)

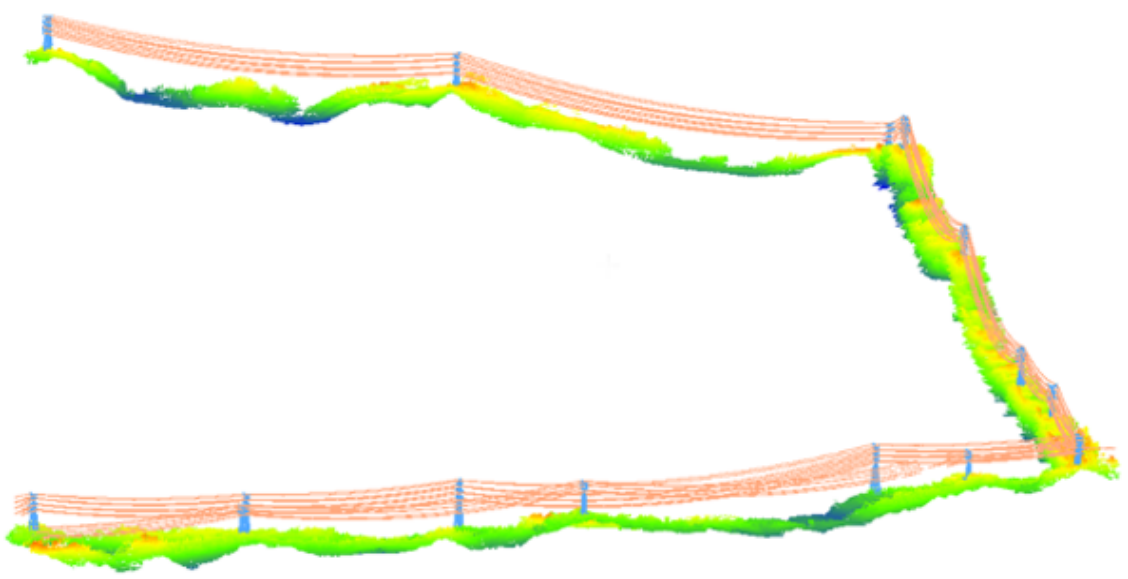

(c)

Figure 17. The main extraction process of $220 \mathrm{kV}$ power lines in a large-scale scene. (a) The original scene of the power lines rendering according to the elevation. (b) The coarse extraction results. The orange points represent the power line points, and the blue points represent the pylon points. (c) The safety distance result of the ground points. The ground points from blue to red indicate that the points are getting closer to the conductors. 
Table 2. Classification accuracy of power line points.

\begin{tabular}{cccc}
\hline & $\mathbf{2 2 0} \mathbf{k V}$ & $\mathbf{5 0 0} \mathbf{k V}$ & $\mathbf{7 5 0} \mathbf{~ V V}$ \\
\hline TP & 373,152 & $1,443,240$ & $1,210,830$ \\
FN & 987 & 2395 & 22,354 \\
FP & 1382 & 7315 & 26,565 \\
Precision & 0.9963 & 0.995 & 0.9785 \\
Recall & 0.9974 & 0.9983 & 0.9818 \\
F-score & 0.9969 & 0.9969 & 0.9802 \\
\hline
\end{tabular}

\subsubsection{The Positioning Accuracy of the Suspension Point}

Figure 18 shows the intuitive extraction effect of suspension points in three different shapes of the pylon of $220 \mathrm{kV}$. The blue points are the pylon points, and the red points are the extracted suspension points. Orange and green points represent power line points in different spans. It can be seen that no matter whether there were power line points on both sides of the pylon (Figure 18a,c) or only one side (Figure 18b), the suspension points at the pylon were extracted accurately. For the pylon with missing power line points on one side, as shown in Figure 18b, we took the vertical plane passing through the center of the pylon as the initial segmentation plane, the suspension points were calculated by the intersection of the conductor and the plane. The spatial positioning accuracy of the suspension points was $0.1968 \mathrm{~m}, 0.1802 \mathrm{~m}, 0.1803 \mathrm{~m}$, for $220 \mathrm{kV}, 500 \mathrm{kV}$ and $750 \mathrm{kV}$, respectively.

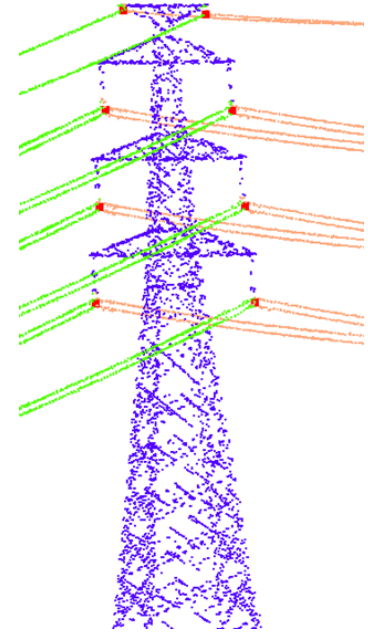

(a)

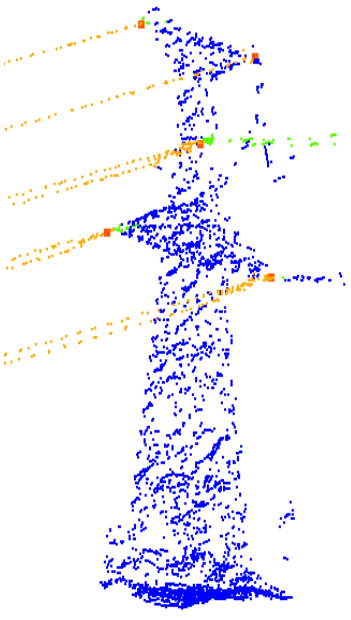

(b)

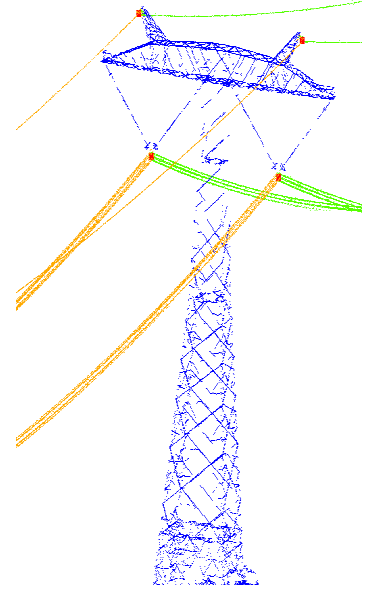

(c)

Figure 18. The suspension points extracted from different shapes of pylons. (a) The extraction result of suspension points at pylon connecting eight power lines. (b) The extraction result of suspension points at pylon with power line missing on one side. (c) The extraction result of suspension points at pylon connecting four power lines.

\subsubsection{The Accuracy and Speed of the Distance Calculation}

To evaluate the performance of the catenary-plane-based safety distance calculation, both the accuracy and efficiency were calculated for power lines of different voltage levels. The results were averaged from the calculation repeated in 15 spans for power lines of different voltage levels. As a comparison, the ground-truth distance was calculated pointby-point.

Table 3 shows the voltage level of the power line and the average number of power line points in one span in the first two columns. The average time consumption of the point-to-point and catenary-plane-based method are listed in the third and fourth columns. 
Not only was the MAE accuracy of the proposed catenary-plane based method less than $0.05 \mathrm{~m}(5 \mathrm{~cm})$ for all the voltage levels, but also the speed of calculation was enhanced thousands of times compared to the ground-truth point-to-point calculation with the resolution of $0.15 \mathrm{~m}$, as shown in the last two columns of Table 3 . It cost about $7 \times 10^{-6} \mathrm{~s}$ per point for surveying the safety distance of power line corridor with a computer equipped with Win $10 \times 64$ system, 16 GB RAM and Intel Core i7-4770 CPU.

Table 3. Average time consuming and MAE in one span.

\begin{tabular}{cccccc}
\hline Voltage Level & Number of Points & Point-Lines Time/s & Point-Points Time/s & MAE/m & Time Ratio \\
\hline $220 \mathrm{kV}$ & 54,492 & 0.334 & 1256.285 & 0.03 & 3760 \\
$500 \mathrm{kV}$ & 31,729 & 0.226 & 948.723 & 0.05 & 4198 \\
$750 \mathrm{kV}$ & 29,977 & 0.287 & 962.924 & 0.02 & 3355 \\
\hline
\end{tabular}

The safety distances between the ground object points and power lines of different voltage levels are shown in Figure 19, where the points are colored from red to blue according to the distance, and the redder the point is, the closer it became to the conductors. With the colored representation of the safety distance, we can intuitively see the position and distance of the potential danger on the ground that influenced the safe operation of the power lines. It can be seen that the safety distance of the power line corridor not only depended on the terrain but also the sagging of the conductor. As shown in Figure 19a and Figure 19c, the regions with high elevation close to the conductors are colored with red. Besides, due to the sagging of the power lines, the vegetation right under the power lines tended to be too close to the power lines, as the many red regions shown in Figure $19 \mathrm{~b}$. With the safety report for all the ground object points, it was convenient to monitor the changes and manage the safety of the power line corridor.

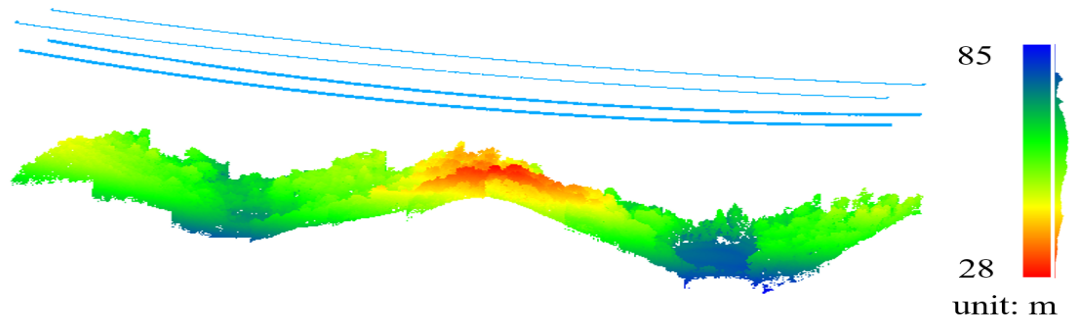

(a)

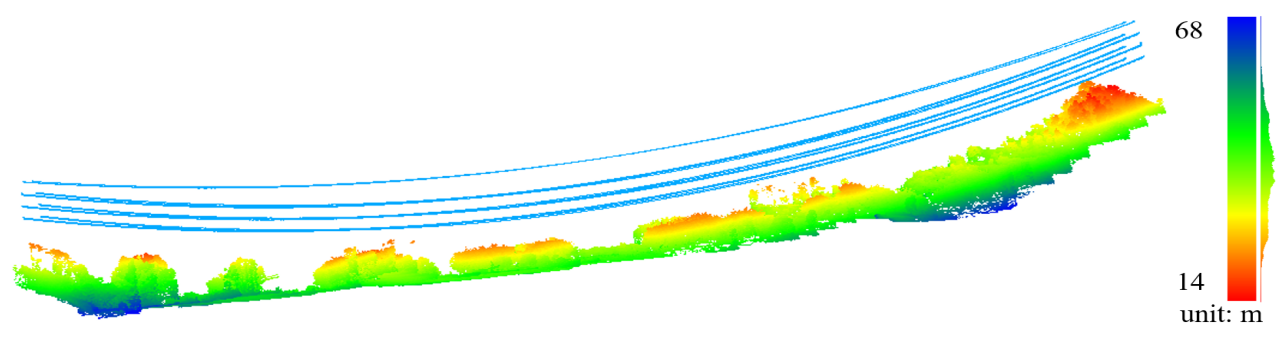

(b)

Figure 19. Cont. 


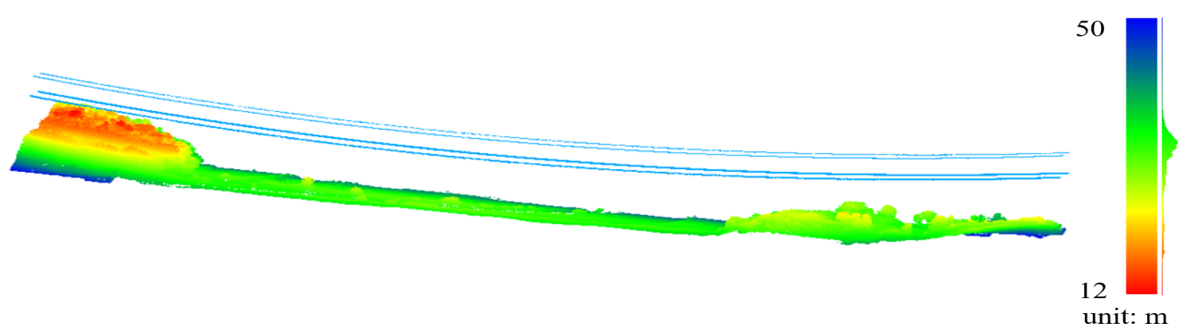

(c)

Figure 19. The safetydistance calculation results of the corridor in three different voltage levels of power lines. The ground points are colored according to the distance from the power lines. The colormap on the right represents the actual distance corresponding to different colors. (a) The safety distance result of $220 \mathrm{kV}$ power line corridor. (b) The safety distance result of $500 \mathrm{kV}$ power line corridor. (c) The safety distance result of $750 \mathrm{kV}$ power line corridor.

\subsubsection{The Influence of Point Density}

To survey the influence of point density on the method in this paper, we carried out some experiments. We down-sampled the point cloud to obtain data with different resolutions of $0.15 \mathrm{~m}$ (original resolution), $0.25 \mathrm{~m}, 0.5 \mathrm{~m}, 1.0 \mathrm{~m}, 1.5 \mathrm{~m}, 2.0 \mathrm{~m}$ and $3.0 \mathrm{~m}$. Tests were performed on these data respectively. As shown in Figure 20, as the resolution decreased from $0.15 \mathrm{~m}$ to $2.0 \mathrm{~m}$, the number of point clouds was reduced, and the time consumption for classifying the point clouds was greatly reduced. As the resolution of the point decreased from $2.0 \mathrm{~m}$ to $3.0 \mathrm{~m}$, the classification accuracy decreased because the interval of point cloud was too large, and the points were very sparse. The geometric feature of pylons became very inconspicuous, which led to classification errors. Since the time consumption of fitting the power lines was little affected by the number of the power line points, the efficiency of the safety distance calculation was slightly improved from $7 \times 10^{-6}$ s to $1 \times 10^{-6}$ s per point with the decrease of the number of power line points.

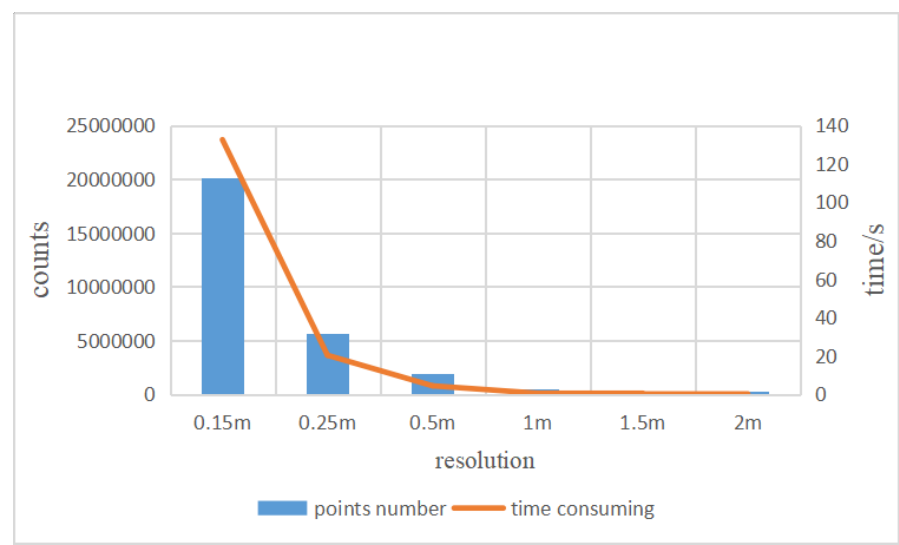

Figure 20. Time consumption for point cloud classification under different point resolution.

\section{Conclusions}

In this paper, an automatic classification and a safety distance calculation method are proposed for UAV LiDAR point cloud of high-voltage power line corridor, which can effectively improve the accuracy and efficiency of power line inspection. Firstly, the histogram analysis is performed on the gridded point cloud to obtain the coarse classification result. According to the characteristics of local linearity of the conductor, the spatial line clustering is then proposed to complete the missing power line points that are caused by the occlusion of trees and underlying waters during the acquisition. The contextual relationship between power lines and pylon is investigated by the grid-based analysis, so that the suspension points of power lines on the pylon are well located. Finally, the catenary 
plane-based simplification of 3D spatial distance calculation between the power lines and ground objects facilitates the survey of the power line corridor.

Experimental results show that the Precision, Recall and F-score for extracting the power lines of $220 \mathrm{kV}, 500 \mathrm{kV}, 750 \mathrm{kV}$ have exceeded $97.85 \%, 98.18 \%$ and $98.02 \%$, respectively. The accuracy of safety distance surveying is $5 \mathrm{~cm}$ for power line corridors of all voltage levels with $0.15 \mathrm{~m}$ resolution of the point cloud, while the speed of surveying is enhanced thousands of times, costing about $7 \times 10^{-3} \mathrm{~s}$ per $10 \mathrm{~m}$ power lines. It is promising to greatly improve both the accuracy and efficiency of surveying the potential hazards of power line corridor.

The catenary plane of the conductor is easily affected by the wind and becomes nonvertical, which may result in an inaccurate measurement of the safety distance for the ground point. In future research, we will study the plane deviation of the power lines under the wind to obtain a more accurate safety distance. Other properties of point clouds, such as intensity, GPSTime and ReturnNumber will be considered to further improve the classification accuracy of point clouds. If the prior pylon coordinate is available, it will be helpful to determine the direction of power lines and to segment the power lines.

Author Contributions: Y.H. and Y.D. conceived and designed the frame of the research; Y.D. performed the experiments; Y.H. and W.S. supervised the research; Y.H. and Y.D. wrote the paper. All authors have read and agreed to the published version of the manuscript.

Funding: This research was funded by the National Science Foundation of China Project (No. 41671419, No. 51208392 and No. 61501334).

Acknowledgments: The authors thank Haitao Ma for providing huge amount of actual drone-based LiDAR data of power lines with different voltage levels for this research.

Conflicts of Interest: The authors declare no conflict of interest.

\section{References}

1. Lou, W.J.; Wu, D.G.; Su, J.; Gu, S.Q.; Jiang, W.D. Discussion on Wind-induced Swing Flashover and Conductor Wind Load of EHV Transmission Lines. High Volt. Eng. 2019, 045, 1249-1255.

2. Chen, C.; Yang, B.; Song, S.; Peng, X.; Huang, R. Automatic Clearance Anomaly Detection for Transmission Line Corridors Utilizing UAV-Borne LIDAR Data. Remote Sens. 2018, 10, 613.

3. Jardini, M.G.M.; Jacobsen, R.M.; Jardini, J.A.; Magrini, L.C.; Beltrame, A.M.K. Information System for the Vegetation Control of Transmission Lines Right-of-way. In Proceedings of the 2007 IEEE Power Tech, Lausanne, Switzerland, 1-5 July 2007.

4. Ahmad, J.; Malik, A.S.; Xia, L.; Ashikin, N. Vegetation encroachment monitoring for transmission lines right-of-ways: A survey. Electr. Power Syst. Res. 2013, 95, 339-352. [CrossRef]

5. Mills, S.; Gerardo Castro, M.P.; Li, Z.; Cai, J.; Hayward, R.; Mejias, L.; Walker, R.A. Evaluation of Aerial Remote Sensing Techniques for Vegetation Management in Power-Line Corridors. IEEE Trans. Geoence Remote Sens. 2010, 48, 3379-3390. [CrossRef]

6. Zhang, R.; Yang, B.; Xiao, W.; Liang, F.; Wang, Z. Automatic Extraction of High-Voltage Power Transmission Objects from UAV Lidar Point Clouds. Remote Sens. 2019, 11, 2600. [CrossRef]

7. Qin, X.; Wu, G.; Ye, X.; Huang, L.; Lei, J. A Novel Method to Reconstruct Overhead High-Voltage Power Lines Using Cable Inspection Robot LiDAR Data. Remote Sens. 2017, 9, 753.

8. Yan, L.; Wu, W.; Li, T. Power transmission tower monitoring technology based on TerraSAR-X products. Proc. SPIE Int. Soc. Opt. Eng. 2011, 10, 82861E.

9. Deng, S.; Zhang, J.; Li, P.; Yang, J. Power line detection from synthetic aperture radar imagery using coherence of co-polarisation and cross-polarisation estimated in the Hough domain. IET Radar Sonar Navig. 2012, 6, 873-880 [CrossRef]

10. Xu, K.; Zhang, X.; Chen, Z.; Wu, W.; Li, T. Risk assessment for wildfire occurrence in high-voltage power line corridors by using remote-sensing techniques: A case study in Hubei Province, China. Int. J. Remote Sens. 2016, 37, 4818-4837. [CrossRef]

11. Kobayashi, Y.; Karady, G.G.; Heydt, G.T.; Olsen, R.G. The Utilization of Satellite Images to Identify Trees Endangering Transmission Lines. IEEE Trans. Power Deliv. 2009, 24, 1703-1709. [CrossRef]

12. Jóźków, G.; Vander Jagt, B.; Toth, C. Experiments with UAS Imagery for Automatic Modeling of Power Line 3D Geometry. Int. Arch. Photogramm. Remote Sens. Spat. Inf. Sci. 2015, 40, 403. [CrossRef]

13. Zhao, H.C.; Lei, J.F.; Wang, X.P.; Zhao, L.; Tian, M.; Cao, W.B.; Yao, H.T.; Cai, B.B. Power line identification algorithm for aerial image in complex background. Bull. Surv. Mapp. 2019, 28-32. [CrossRef]

14. Jadin, M.S.; Ghazali, K.H.; Taib, S. Thermal condition monitoring of electrical installations based on infrared image analysis. In Proceedings of the Electronics, Communications \& Photonics Conference, Riyadh, Saudi Arabia, 27-30 April 2013. 
15. Zhang, W.F.; Peng, X.; Chen, R.; Chen, C.; Deng, Y.; Qian, J.; Xu, W. Intelligent Diagnostic Techniques of Abnormal Heat Defect in Transmission Lines Based on Unmanned Helicopter Infrared Video. Power Syst. Technol. 2014, 38, 1334-1338.

16. Nardinocchi, C.; Balsi, M.; Esposito, S. Fully Automatic Point Cloud Analysis for Powerline Corridor Mapping. IEEE Trans. Geosci. Remote Sens. 2020, 58, 8637-8648. [CrossRef]

17. Chen, C.; Mai, X.M.; Song, S.; Peng, X.Y.; Xu, W.X.; Wang, K. Automatic power line extraction method for airborne laser point cloud data. Geomat. Inf. Sci. Wuhan Univ. 2015, 40, 1600-1605.

18. Jung, J.; Che, E.; Olsen, M.J.; Shafer, K.C. Automated and efficient powerline extraction from laser scanning data using a voxel-based subsampling with hierarchical approach. ISPRS J. Photogramm. Remote Sens. 2020, 163, 343-361. [CrossRef]

19. Husain, A.; Vaishya, R.C. An automated method for power line points detection from terrestrial LiDAR data. In Emerging Technologies in Data Mining and Information Security; Springer: Berlin, Germany, 2019; pp. 459-472.

20. Nasseri, M.H.; Moradi, H.; Nasiri, S.M.; Hosseini, R. Power Line Detection and Tracking Using Hough Transform and Particle Filter. In Proceedings of the 2018 6th RSI International Conference on Robotics and Mechatronics (IcRoM), Tehran, Iran, 23-25 October 2018.

21. Zheng, L.; Yi, R. Fault diagnosis system for the inspection robot in power transmission lines maintenance. In Proceedings of the 2009 International Conference on Optical Instruments and Technology: Optoelectronic Imaging and Process Technology, Shanghai, China, 19-22 October 2009.

22. Guan, H.; Yu, Y.; Li, J.; Ji, Z.; Zhang, Q. Extraction of power-transmission lines from vehicle-borne lidar data. Int. J. Remote Sens. 2016, 37, 229-247. [CrossRef]

23. Xiaowei, Y.; Juha, H.; Mika, K.; Kimmo, N.; Kirsi, K.; Mikko, V.; Ville, K.; Harri, K.; Markus, H.; Eija, H. Comparison of Laser and Stereo Optical, SAR and InSAR Point Clouds from Air- and Space-Borne Sources in the Retrieval of Forest Inventory Attributes. Remote Sens. 2015, 7, 15933-15954.

24. Blistan, P.; Kovanič, L.; Zelizňaková, V.; Palková, J. Using UAV photogrammetry to document rock outcrops. Acta Mont. Slovaca 2016, 21, 154-161.

25. Sithole, G.; Vosselman, G. Experimental comparison of filter algorithms for bare-Earth extraction from airborne laser scanning point clouds. ISPRS J. Photogramm. Remote Sens. 2004, 59, 85-101. [CrossRef]

26. Zeybek, M.; İsmail, Ş. Point cloud filtering on UAV based point cloud. Measurement 2019, 133, 99-111. [CrossRef]

27. Huang, H.; Brenner, C.; Sester, M. A generative statistical approach to automatic 3D building roof reconstruction from laser scanning data. ISPRS J. Photogramm. Remote Sens. 2013, 79, 29-43. [CrossRef]

28. Aicardi, I.; Dabove, P.; Lingua, A.M.; Piras, M. Integration between TLS and UAV photogrammetry techniques for forestry applications. IForest Biogeosci. For. 2016, 10, e1-e7. [CrossRef]

29. Garrido, M.; Paraforos, D.; Reiser, D.; Vázquez Arellano, M.; Griepentrog, H.; Valero, C. 3D Maize Plant Reconstruction Based on Georeferenced Overlapping LiDAR Point Clouds. Remote Sens. 2015, 7, 17077-17096. [CrossRef]

30. Ituen, I.; Sohn, G. The Way Forward: Advances in Maintaining Right-of-Way of Transmission Lines. Geomatica 2010, 64, 451-462.

31. Wang, Y.; Chen, Q.; Liu, L.; Zheng, D.; Li, C.; Li, K.. Supervised Classification of Power Lines from Airborne LiDAR Data in Urban Areas. Remote Sens. 2017, 9,771.

32. Pu, S.; Xie, L.; Ji, M.; Zhao, Y.; Liu, W.; Wang, L.; Zhao, Y.; Yang, F.; Qiu, D. Real-time powerline corridor inspection by edge computing of UAV Lidar data. Int. Arch. Photogramm. Remote Sens. Spat. Inf. Sci. 2019, 4213, 547-551. [CrossRef]

33. Liu, Y.; Li, Z.; Hayward, R.; Walker, R.A.; Jin, H. Classification of Airborne LIDAR Intensity Data Using Statistical Analysis and Hough Transform with Application to Power Line Corridors. In Proceedings of the 2009 Digital Image Computing: Techniques and Applications, Melbourne, VIC, Australia, 1-3 December 2009; pp. 462-467.

34. Melzer, T.B.C. Extraction and Modeling of Power Lines from ALS Point Clouds. In Proceedings of the Workshop, 28th Workshop of the Austrian Association for Pattern Recognition (OAGM), Salzburg, Austria, 22-23 May 2004; pp. 47-54.

35. Yu, J.; Mu, C.; Feng, Y.M.; Dou, Y.J. Study on power line extraction from airborne LiDAR point cloud data. Geomat. Inf. Sci. Wuhan Univ. 2011, 36, 1275-1279.

36. Wang, P.H.; Xi, X.H.; Wang, C.; Xia, S.B. Study on power line fast extraction based airborne LiDAR data. Sci. Surv. Mapp. 2017, 42, 154-158.

37. Awrangjeb, M. Extraction of Power Line Pylons and Wires Using Airborne LiDAR Data at Different Height Levels. Remote Sens.2019, 11, 1798-1822. [CrossRef]

38. Ma, W.F.; Wang, C.; Wang, J.; Zhou, J.; Ma, Y. Extraction of power lines from laser point cloud based on residual clustering method. Acta Geod. Et Cartogr. Sin. 2020, 49, 883.

39. Jwa, Y.; Sohn, G.; Kim, H.B. Automatic 3D powerline reconstruction using airborne lidar data. Int. Arch. Photogramm. Remote Sens. 2009, 38, W8.

40. Chen, C.; Peng, X.Y.; Song, S.; Wang, K.; Qian, J.J.; Yang, B.S. Safety Distance Diagnosis of Large Scale Transmission Line Corridor Inspection Based on LiDAR Point Cloud Collected With UAV. Power Syst. Technol. 2017, 041, 2723-2730.

41. Blomley, R.; Jutzi, B.; Weinmann, M. Classification of Airborne Laser Scanning Data Using Geometric Multi-Scale Features and Different Neighbourhood Types. ISPRS Ann. Photogramm. Remote Sens. Spat. Inf. 2016, III-3, 169-176. [CrossRef]

42. Liang, C.; Tong, L.; Wang, Y.; Li, M. Extraction of Urban Power Lines from Vehicle-Borne LiDAR Data. Remote Sens. 2014, 6, 3302-3320. 
43. Bo, G.; Huang, X.; Fan, Z.; Sohn, G. Classification of airborne laser scanning data using JointBoost. ISPRS J. Photogramm. Remote Sens. 2015, 100, 71-83.

44. Zhou, R.Q.; Xu, Z.H.; Peng, C.G.; Zhang, F.; Jiang, W.S. A JointBoost-based classification method of high voltage transmission corridor from airborne LiDAR point cloud. Sci. Surv. Mapp. 2019, 44, 21-27.

45. Kim, H.B.; Sohn, G. Point-based Classification of Power Line Corridor Scene Using Random Forests. Photogramm. Eng. Remote Sens. 2013, 79, 821-833. [CrossRef]

46. Li, Q.; Chen, Z.; Hu, Q. A Model-Driven Approach for 3D Modeling of Pylon from Airborne LiDAR Data. Remote Sens. 2015, 7, 11501-11524. [CrossRef]

47. Peng, X.Y.; Song, S.; Qian, J.J.; Chen, C.; Wang, K.; Yang, Y.Q.; Zheng, X.G. Research on Automatic Positioning Algorithm of Power Transmission Towers Based on UAV LiDAR. Power Syst. Technol. 2017, 41, 3670-3677.

48. Liu, Y.; Yang, B.S.; Liang, F.X. An automatically recognizing method for transmission towers from ALS point cloud. Bull. Surv. Mapp. 2019, 01, 34-38.

49. Hongyun, S.; Tao, G.; Di, W.; Shichun, W.; Jian, Z.; Xin, L.; Xin, L. Power line suspension point location method based on laser point cloud. Laser Technol. 2020, 44, 364-370.

50. Toschi, I.; Morabito, D.; Grilli, E.; Remondino, F.; Carlevaro, C.; Cappellotto, A.; Tamagni, G.; Maffeis, M. Cloud-Based Solution for Nationwide Power Line Mapping. ISPRS Int. Arch. Photogramm. Remote Sens. Spat. Inf. Sci. 2019, XLII-2/W13, 119-126. [CrossRef]

51. Xu, B.; Liu, Z.J.; Wang, J. Extraction and Security Detection of Power Line Based on Laser Point Cloud Data. Laser J. 2017, $38,48-51$.

52. Jwa, Y.; Sohn, G. A multi-level span analysis for improving 3D power-line reconstruction performance using airborne laser scanning data. In Proceedings of the ISPRS Technical Commission III Symposium PCV 2010—Photogrammetric Computer Vision and Image Analysis, Saint-Mandé, France, 1-3 September 2010.

53. Redding, N.J. Implicit polynomials, orthogonal distance regression, and the closest point on a curve. IEEE Trans. Pattern Anal. Mach. Intell. 2000, 22, 191-199. [CrossRef]

54. Silver, E.A.; Bischak, D.P.; Silveira, G.J.C.D. An efficient method for calculating the minimum distance from an operating point to a specific (hyperbolic) efficient frontier. IMA J. Manag. Math. 2009, 20, 251-261. [CrossRef]

55. William, H. Numerical recipes in FORTRAN. The art of scientific computing. IEEE Concurr. 1997, 6, 79.

56. Ostu, N. A threshold selection method from gray-histogram. IEEE Trans. Syst. Man Cybern. 1975, 11, $285-296$.

57. Foley, J.D.; Dam, A.V.; Feiner, S.; Hughes, J.F. Computer Graphics: Principles and Practice, 2nd ed.; Addison-Wesley Professional: Boston, MA, USA, 1996

58. Wu, L.F.; Chen, Y.P.; Chen, Y.H. Algorithms on calculating minimum distance between point and spatial parametric curves. Mach. Des. Manuf. 2011, 15-17.

59. Jwa, Y.; Sohn, G. A Piecewise Catenary Curve Model Growing for 3D Power Line Reconstruction. Photogramm. Eng. Remote Sens. 2012, 78, 1227-1240. [CrossRef] 